\title{
OPTIMAL MULTILEVEL METHODS FOR GRADED BISECTION GRIDS
}

\author{
LONG CHEN *, RICARDO H. NOCHETTO †, AND JINCHAO XU ‡
}

\begin{abstract}
We design and analyze optimal additive and multiplicative multilevel methods for solving $H^{1}$ problems on graded grids obtained by bisection. We deal with economical local smoothers: after a global smoothing in the finest mesh, local smoothing for each added node during the refinement needs to be performed only for three vertices - the new vertex and its two parent vertices. We show that our methods lead to optimal complexity for any dimensions and polynomial degree. The theory hinges on a new decomposition of bisection grids in any dimension, which is of independent interest and yields a corresponding decomposition of spaces. We use the latter to bridge the gap between graded and quasi-uniform grids, for which the multilevel theory is well-established.
\end{abstract}

Key words. subspace correction method, local mesh refinement, bisection method, multilevel preconditioning, BPX, V-cycle multigrid, optimal complexity

AMS subject classifications. 65M55, 65N55, 65N22, 65F10.

1. Introduction. Adaptive methods are now widely used in scientific and engineering computation to optimize the relation between accuracy and computational labor (degrees of freedom). Standard adaptive finite element methods (AFEM) based on local mesh refinement can be written as loops of the form

SOLVE $\rightarrow$ ESTIMATE $\rightarrow$ MARK $\rightarrow$ REFINE.

The module ESTIMATE determines a posteriori error estimators; we refer to [58]. The module MARK selects elements with largest error indicators and is critical for convergence and optimality of AFEM. Neither of these two procedures plays a role in the present discussion. The module REFINE refines all marked elements and perhaps a few more to keep mesh conformity. Of all possible refinement strategies, we are interested in bisection, a popular, elegant, and effective procedure for refinement in any dimension [48, 8, 32, 33, 55, 3, 49, 54]. Our goal is to design optimal multilevel solvers that constitute the core of procedure SOLVE, and analyze them within the framework of highly graded meshes created by bisection, from now on called bisection grids.

It is important to realize that having optimal solvers in SOLVE is crucial for the theory and practice of AFEM. Convergence of adaptive loops (1.1) for dimension $d>1$ started with the seminal work of Dörfler [26]. This was followed by Morin, Nochetto and Siebert [40, 41], who realized the role of data oscillation, and Mekchay and Nochetto [36], who proved a contraction property for AFEM for general elliptic PDEs. More recently, Binev, Dahmen, and DeVore [10] proved quasi-optimal cardinality for a modified algorithm including coarsening. Stevenson [53] was able to remove coarsening that still needs an artificial inner loop. The most standard AFEM has been later examined by Cascón, Kreuzer, Nochetto and Siebert [18], who have proved a contraction property and quasi-optimal cardinality. We refer to Nochetto, Siebert and Veeser [42] for an introduction to the theory of adaptive finite element

*Department of Mathematics, University of California at Irvine, CA, 92697 (chenlong@math.uci.edu). The first author was supported in part by NSF Grant DMS-0505454, DMS-0811272, and in part by NIH Grant P50GM76516 and R01GM75309.

$\dagger$ Department of Mathematics, University of Maryland, College Park, MD, 20742 (rhn@math. umd. edu). The second author was supported in part by NSF Grant DMS-0505454 and DMS-0807811.

$\ddagger$ The School of Mathematical Science, Peking University, and Department of Mathematics, Pennsylvania State University, University Park, PA 16801 (xu@math.psu.edu). The third author was supported in part by NSF DMS-0609727, DMS 0915153, NSFC-10528102 and Alexander von Humboldt Research Award for Senior US Scientists. 
methods. The gap to obtaining optimal complexity is precisely having optimal solvers and storage for adaptive bisection grids - the topic of this paper.

We consider a nested family of finite element spaces obtained by local mesh refinement:

$$
\mathcal{V}_{0} \subseteq \mathcal{V}_{1} \subseteq \cdots \subseteq \mathcal{V}_{J}=\mathcal{V}
$$

A standard multilevel method contains a smoothing step on the spaces $\mathcal{V}_{j}, j=0, \ldots, J$. For graded grids obtained by AFEM, it is possible that $\mathcal{V}_{j}$ results from $\mathcal{V}_{j-1}$ by just adding few, say one, basis function. Thus smoothing on both $\mathcal{V}_{j}$ and $\mathcal{V}_{j-1}$ leads to a lot of redundancy. If we let $N$ be the number of unknowns in the finest space $\mathcal{V}$, then the complexity of smoothing can be as bad as $\mathcal{O}\left(N^{2}\right)$. To achieve optimal complexity $\mathcal{O}(N)$, the smoothing in each space $\mathcal{V}_{j}$ must be restricted to the new unknowns and their neighbors. Such methods are referred to as local multilevel methods $[17,4]$. Performing the smoothing only on the newly added nodes, the most extreme choice, gives rise to the hierarchical basis (HB) method $[66,6]$.

Since the literature on local multilevel methods is abundant, we restrict ourselves to describing the papers most relevant to graded meshes. Brandt [17] proposed the multilevel adaptive technique (MLAT) and further studied it in [4]. McCormick and collaborators [34, 35] developed the fast adaptive composite grid (FAC) method, which requires exact solvers on subdomains that are partitioned by uniform grids; hence mesh adaptivity is achieved via superposition of tensor-product rectangular grids. Rivara [47] and Mitchell [37, 38] developed local multigrid methods on adaptive triangular grids obtained by longest edge bisection and newest vertex bisection, respectively, for $d=2$. Also for $d=2$, Bank, Sherman and Weiser [7] proposed the red-green refinement strategy, which was implemented in the wellknown piecewise linear triangular multigrid software package (PLTMG) of Bank [5]. For the resulting locally refined grids, Bank, Dupont and Yserentant [6] developed HB multigrid, which is a variant of the HB preconditioner developed earlier by Yserentant [65]. They proved that the hierarchical basis methods are nearly optimal (up to a logarithmic factor on the number of elements) for $d=2$, and suboptimal for $d>2$. Bramble, Pasciak and Xu [15] proposed the BPX preconditioner on both quasi-uniform grids and locally refined grids, and showed that it is nearly optimal for any dimension $d$. Oswald [44] was able to remove the logarithmic factor, and thus proved optimal complexity of BPX preconditioner and established a similar result on locally refined grids in [45]. Bramble and Pasciak [14] proved the optimality of multilevel algorithms including BPX preconditioner and V-cycle multigrid methods on quasi-uniform and locally refined grids. Dahmen and Kunoth [24] proved optimal complexity of BPX, for graded meshes created by red-green refinement for $d=2$; see also Bornemann and Yserentant [13] for a simpler approach using a K-functor. Griebel [27, 30] developed multilevel methods on adaptive sparse grids. Xu [61] introduced a unified abstract framework for the various multilevel methods based on the method of subspace corrections, which is the approach we pursue in this paper. More recently, $\mathrm{Wu}$ and Chen [60] analyzed multigrid methods using Gauss-Seidel type smoothers on bisection grids generated by newest vertex bisection for $d=2$. Finally, Aksoylu and Holst [2] extended the optimality results of [24] to a practical, local red-green refinement procedure for $d=3$ due to Bornemann, Erdmann, and Kornhuber [11].

We now summarize our four main contributions and place them in context.

- First we present a novel decomposition of bisection grids. Roughly speaking, for any triangulation $\mathcal{T}_{N}$ constructed from $\mathcal{T}_{0}$ by $N$ bisections, we can write

$$
\mathcal{T}_{N}=\mathcal{T}_{0}+\mathcal{B}, \quad \mathcal{B}=\left\{b_{1}, b_{2}, \cdots, b_{N}\right\}
$$

where $\mathcal{B}$ denotes an ordered sequence of $N$ elementary bisections $b_{i}$. Each $b_{i}$ is restricted to a local region, the star of the newly created vertex, and the corresponding local grid 
is thus quasi-uniform. This decomposition induces a space decomposition $\left\{\mathcal{V}_{i}\right\}_{i=1}^{N}$ of the underlying subspace of continuous piecewise linear functions over $\mathcal{T}_{N}$. Moreover, this decomposition serves as a general bridge to transfer results from quasi-uniform grids to graded bisection grids and has some intrinsic interest. For example, it is this geometric structure of bisection grids that motivates the new efficient implementation of multilevel methods developed by Chen and Zhang [22] for $d=2$ and by Chen [19] for $d=3$, which avoids dealing with the tree structure of the mesh and hinges on coarsening from the finest mesh $\mathcal{T}_{N}$ to find (1.2). Such a grid decomposition may or may not coincide with the one giving rise to $\mathcal{T}_{N}$ via AFEM, but it does not matter.

We stress that the popularity of multilevel methods among practitioners is somehow hampered by their complicated data structures to store and access the hierarchical grid structure. Efficient multilevel algorithms developed in [22] and [19] require only minimal bookkeeping (recording the two parent nodes for each new bisection node) and a simple data structure (only the finest grid must be stored instead of the whole refinement tree). They rely on a clever coarsening algorithm for bisection grids and exhibit both optimal storage and complexity. This paper provides a theoretical basis for these methods and establishes their optimality.

- Second, we introduce and analyze economical local smoothers which reduce the complexity of the resulting multilevel methods. In fact, besides a smoothing in the finest grid $\mathcal{T}_{N}$, the local smoothing (or local relaxation) associated with each bisection $b_{i}$ is just performed for the newly created vertex and its two parent vertices. This implies that $\operatorname{dim} \mathcal{V}_{i}=3$, whence the total complexity is proportional to the size of the linear system with a relatively small constant.

From the algorithmic point of view, our algorithm is different from the traditional local multigrid, which requires smoothing for the new vertex and all neighboring vertices (and degrees of freedom for quadratic or higher order elements) but no additional smoothing in the finest grid. In one iteration of V-cycle or BPX preconditioner, our algorithm requires less operations than the traditional one. For example, for linear elements, treating the cost of the smoothing on one node as a unit, our algorithm requires $4 N$ operations while the traditional one may need $k N$ operations, where $k$ is the number of neighboring nodes surrounding a node. For bisection grids, the average of $k$ is 5 for $d=2$ and could be as high as 10 for $d=3$. Furthermore our algorithm is easier to implement, especially for higher order elements. The smoothing on the finest grid can be easily realized using Jacobi or Gauss-Seidel methods since the matrix is given while the multilevel smoothing only involves linear elements and three points. Note that the new vertex and two parents vertices are minimal information needed, in any geometric multigrid method, to construct the restriction and prolongation operators.

Our three-point smoother, inspired by an idea of Stevenson [52] for wavelets, can be also thought of as an economical way to stabilize the hierarchical basis (HB) methods for $d>2$, which otherwise are known to be suboptimal. Other stabilization attempts for the HB methods can be found in [56, 57]. We stress that the smoothing in the finest grid, which follows from the principle of auxiliary space method [62], plays an important role in the stabilization.

- Third, we provide an analysis without the so-called nested refinement assumption:

$$
\Omega_{J} \subseteq \Omega_{J-1} \subseteq \cdots \subseteq \Omega_{0}=\Omega
$$

with each subdomain $\Omega_{j}$ being made of all the new elements created at the $j$-th level which, therefore, were not present earlier. This also implies that all elements contained in subdomain $\Omega_{j}$ possess the same generation $j$ and a comparable size. The grid corresponding to 
$\Omega_{j}$ is thus quasi-uniform, and local multigrid methods can be analyzed using a truncated $L^{2}$-projection $[15,14]$.

A natural question then arises: how can we apply existing theories to bisection grids which may not obey the nested refinement assumption (1.3)? For fully additive multilevel methods, e.g. the original BPX preconditioner, the ordering does not matter in the implementation and analysis and thus we can always assume (1.3) holds. For multiplicative methods (e.g. V-cycle multigrid or additive multilevel method with multiplicative smoothers), further work needs to be done to apply the existing theories.

One approach is to use the relation between the additive and the multiplicative method $[29,16,23]$. Roughly speaking, if additive preconditioner leads to a uniformly bounded condition number for the preconditioned system, then the multiplicative methods is convergent with a rate depends at most on $J$ the number of levels. For quasi-uniform grids, $J \approx|\log h|$ which is an acceptable factor in practice. For bisection grids, however, the level $J$ could be $\mathcal{O}(N)$ in the worst scenario and thus this estimate is not optimal.

When the tree structure of the local mesh refinement is available, one could reconstruct a virtual local mesh refinement hierarchy by grouping all elements with the same generation into one level such that the assumption (1.3) holds [12,11,31] and implement multigrid algorithms on this virtual nested refinement. However, these levels increase dynamically within AFEM and must be updated for every loop (1.1). Consequently, reconstructing a virtual refinement hierarchy entails implementing suitable bookkeeping data structures which might compromise optimal storage and thus optimality.

The new algorithms [19, 22], as well as [1], show that multilevel methods retain optimality even when the nested refinement assumption (1.3) is violated or elements with disparate sizes are grouped together into one refinement patch. This paper provides an alternative approach to analyze these more flexible algorithms.

- Four, we provide a unified framework for analysis of multilevel methods on graded bisection grids, which is valid for any dimension $d$, any polynomial degree, and minimal regularity. We should point out that $\mathrm{Wu}$ and Chen [60] have analyzed multigrid methods for bisection grids and $d=2$ without the nested refinement assumption (1.3). Their proof of uniform convergence relies on the specific geometric structure of bisection grids for $d=2$, and its extension to $d>2$ seems rather difficult. Our approach below is conceptually simpler than [60], applies to any dimension $d$ as well as general smoothers, rather than just Gauss-Seidel, and extends to BPX preconditioners. Our analysis, carried out in Section 2.4, hinges on three basic properties: the contraction property (2.6) of the local (inexact) smoother, the stability bound (1.6) of the subspace decomposition, and the strengthened Cauchy-Schwarz inequality (1.7). The proofs of (1.6) and (1.7) are the core of this paper and are given in Section 4. They heavily rely on the decomposition of bisection grids discussed earlier in Section 3.

In the rest of the introduction, we briefly present the model problem and outline our approach. Let $\Omega \subset \mathbb{R}^{d}, d \geq 2$ be a polyhedral domain, and consider the Dirichlet form

$$
a(u, v):=\int_{\Omega} \nabla u \cdot \nabla v \mathrm{~d} x .
$$

Given a (graded) triangulation $\mathcal{T}$ of $\Omega$, we choose the finite element space $\mathcal{V}:=\left\{v \in H_{0}^{1}(\Omega)\right.$ : $\left.v\right|_{\tau} \in \mathcal{P}_{m}(\tau)$, for all $\left.\tau \in \mathcal{T}\right\}$, where $\mathcal{P}_{m}(\tau)$ is the space of polynomials on $\tau$ of degree $\leq m$, with $m \geq 1$, and let $u \in \mathcal{V}$ be the finite element solution of the second order elliptic equation

$$
(A u, v):=a(u, v)=\langle f, v\rangle \quad \text { for all } v \in \mathcal{V},
$$

where $f \in H^{-1}(\Omega), A: \mathcal{V} \rightarrow \mathcal{V}$ is the discrete Laplacian, and $\langle\cdot, \cdot\rangle$ is the duality pair. For 
ease of exposition, we let $\Omega$ be partitioned exactly into a bisection grid $\mathcal{T}$, which is shape regular and conforming, and consider the Laplace operator.

Our analysis can be generalized to variable coefficients with small variations, in which case the contraction factor will depend on the variations of the coefficients. For variable coefficients, possibly with large jumps across interelement boundaries, we refer to [21]: using local multigrid as a preconditioner of the conjugate gradient method yields a robust method with respect to both the mesh size and the size of jump discontinuities of the coefficients.

We now briefly discuss our approach to multilevel methods. Let $\left\{\phi_{p}\right\}_{p \in \Lambda}$ be the canonical basis functions of the space $\mathcal{V}$ and let $\mathcal{V}_{p}=\operatorname{span}\left\{\phi_{p}\right\}$ with $\operatorname{dim} \mathcal{V}_{p}=1$ for $p \in \Lambda$; thus $\mathcal{V}=\operatorname{span}\left\{\phi_{p}\right\}_{p \in \Lambda}$. Let $\mathcal{V}_{i} \subset \mathcal{V}$ with $\operatorname{dim} \mathcal{V}_{i}=3$ be the space of piecewise linear functions spanned by the newest vertex added by each elementary bisection $b_{i}$ and its two parents vertices, for $i=1, \cdots, N$, and let $\mathcal{V}_{0}$ be the coarsest space of piecewise linear elements over $\mathcal{T}_{0}$. We then have the space decomposition

$$
\mathcal{V}=\sum_{p \in \Lambda} \mathcal{V}_{p}+\sum_{i=0}^{N} \mathcal{V}_{i}
$$

for which we shall prove the following two key properties:

- Stable Decomposition: For any $v \in \mathcal{V}$, there exist $v_{p} \in \mathcal{V}_{p}, p \in \Lambda$, and $v_{i} \in \mathcal{V}_{i}, i=$ $0, \cdots, N$ such that $v=\sum_{p \in \Lambda} v_{p}+\sum_{i=0}^{N} v_{i}$ and

$$
\sum_{p \in \Lambda} h_{p}^{-2}\left\|v_{p}\right\|^{2}+\sum_{i=0}^{N} h_{i}^{-2}\left\|v_{i}\right\|^{2} \lesssim|v|_{1}^{2} .
$$

- Strengthened Cauchy-Schwarz (SCS) Inequality: For any $u_{i}, v_{i} \in \mathcal{V}_{i}, i=0, \cdots, N$, we have

$$
\left|\sum_{i=0}^{N} \sum_{j=i+1}^{N} a\left(u_{i}, v_{j}\right)\right| \lesssim\left(\sum_{i=0}^{N}\left|u_{i}\right|_{1}^{2}\right)^{1 / 2}\left(\sum_{i=1}^{N}\left|v_{i}\right|_{1}^{2}\right)^{1 / 2} .
$$

Hereafter $h_{p}$ or $h_{i}$ represent local meshsizes corresponding to $\mathcal{V}_{p}$ or $\mathcal{V}_{i}$, respectively. With the help of (1.6) and (1.7), derived in Section 4, we are able to obtain optimal multilevel methods including BPX preconditioner and V-cycle multigrid methods for solving the algebraic system (1.4) over graded bisection grids. We prove convergence of these methods in Section 2.4.

We use standard Sobolev space notation: $\|\cdot\|$ denotes the $L^{2}$-norm and $|\cdot|_{1}$ the $H^{1}$ semi-norm, which is a norm on $H_{0}^{1}(\Omega)$. We write $x \lesssim y$ to indicate $x \leq C y$, with constant $C$ independent of problem size $N$ and functions $v \in \mathcal{V}$, as well as $x \approx y$ to mean $x \lesssim y$ and $y \lesssim x$.

The rest of this paper is organized as follows. In Section 2, we review the subspace correction method and provide abstract convergence analysis based on three assumptions: (1.6), (1.7), and (2.6) below. In Section 3, we discuss bisection methods and present the crucial decomposition of bisection grids. In Section 4, we first obtain a space decomposition based on the decomposition of bisection grids and next prove (1.6) and (1.7). Finally, in Section 5 we summarize optimal complexity results for both local BPX-preconditioner and $\mathrm{V}$-cycle multigrid, which are valid for inexact local solvers which induce the contraction (2.6).

2. The Method of Subspace Corrections. Discretization of partial differential equations often leads to linear algebraic equations of the form

$$
A u=f,
$$


where $A \in \mathbb{R}^{N \times N}$ is a sparse matrix and $f \in \mathbb{R}^{N}$. In this section, we give some general and basic results that will be used in later sections to construct efficient multilevel iterative methods (such as multigrid methods) for (2.1) resulting from finite element discretizations of elliptic partial differential equations. The presentation in this section follows closely to $\mathrm{Xu}$ [61] with simplified analysis.

2.1. Iterative Methods. A basic linear iterative method for $A u=f$ can be written in the following form

$$
u^{k+1}=u^{k}+B\left(f-A u^{k}\right),
$$

starting from an initial guess $u^{0} \in \mathcal{V} ; B$ is called iterator. If $A=\left(a_{i j}\right) \in \mathbb{R}^{N \times N}$ is split into diagonal, lower and upper triangular parts, namely $A=D+L+U$, then two classical examples are the Jacobi method $B=D^{-1}$ and the Gauss-Seidel method $B=(D+L)^{-1}$.

The art of constructing efficient iterative methods lies on the design of $B$ which captures the essential information of $A^{-1}$ and its action is easily computable. In this context the notion of "efficiency" entails two essential requirements:

- One iteration requires a computational effort proportional to the number of unknowns.

- The rate of convergence is well below 1 and independent of the number of unknowns.

The approximate inverse $B$, when it is SPD, can be used as a preconditioner for Conjugate Gradient (CG) method. The resulting method, known as preconditioned conjugate gradient method (PCG), admits the following error estimate in terms of the condition number $\kappa(B A)=\lambda_{\max }(B A) / \lambda_{\min }(B A)$

$$
\frac{\left\|u-u^{k}\right\|_{A}}{\left\|u-u^{0}\right\|_{A}} \leq 2\left(\frac{\sqrt{\kappa(B A)}-1}{\sqrt{\kappa(B A)}+1}\right)^{k} \quad(k \geq 1) ;
$$

$B$ is called preconditioner. A good preconditioner should have the properties that the action of $B$ is easy to compute and that $\kappa(B A)$ is significantly smaller than $\kappa(A)$.

2.2. Space Decomposition and Method of Subspace Corrections. In the spirit of divide and conquer, we decompose the space $\mathcal{V}=\sum_{i=0}^{f} \mathcal{V}_{i}$ as the summation of subspaces $\mathcal{V}_{i} \subset \mathcal{V} ;\left\{\mathcal{V}_{i}\right\}_{i=0}^{J}$ is called a space decomposition of $\mathcal{V}$. Since $\sum_{i=0}^{J} \mathcal{V}_{i}$ is not necessarily a direct sum, decompositions of $u \in \mathcal{V}$ of the form $u=\sum_{i=0}^{J} u_{i}$ are in general not unique. The original problem (2.1) can thus be split into sub-problems in each $\mathcal{V}_{i}$ with smaller size which are relatively easier to solve.

Throughout this paper, we use the following operators, for $i=0,1, \ldots, J$ :

- $Q_{i}: \mathcal{V} \rightarrow \mathcal{V}_{i}$ the projection in the inner product $(\cdot, \cdot)$;

- $I_{i}: \mathcal{V}_{i} \rightarrow \mathcal{V}$ the natural inclusion which is often called prolongation;

- $P_{i}: \mathcal{V} \rightarrow \mathcal{V}_{i}$ the projection in the inner product $(\cdot, \cdot)_{A}=(A \cdot, \cdot)$;

- $A_{i}: \mathcal{V}_{i} \rightarrow \mathcal{V}_{i}$ the restriction of $A$ to the subspace $\mathcal{V}_{i}$;

- $R_{i}: \mathcal{V}_{i} \rightarrow \mathcal{V}_{i}$ an approximation of $A_{i}^{-1}$ (often known as smoother);

- $T_{i}: \mathcal{V} \rightarrow \mathcal{V}_{i} \quad T_{i}=R_{i} Q_{i} A=R_{i} A_{i} P_{i}$.

It is easy to verify the relation $Q_{i} A=A_{i} P_{i}$ and $Q_{i}=I_{i}^{t}$ with $\left(I_{i}^{t} u, v_{i}\right):=\left(u, I_{i} v_{i}\right)$. The operator $I_{i}^{t}$ is often called restriction. If $R_{i}=A_{i}^{-1}$, then we have an exact local solver and $R_{i} Q_{i} A=P_{i}$. With slightly abused notation, we still use $T_{i}$ to denote the restriction $\left.T_{i}\right|_{\mathcal{V}_{i}}: \mathcal{V}_{i} \rightarrow \mathcal{V}_{i}$ and $T_{i}^{-1}=\left(\left.T_{i}\right|_{\mathcal{V}_{i}}\right)^{-1}: \mathcal{V}_{i} \rightarrow \mathcal{V}_{i}$.

For a given residual $r \in \mathcal{V}$, we let $r_{i}=Q_{i} r=I_{i}^{t} r$ denote the restriction of the residual to the subspace $\mathcal{V}_{i}$ and solve the residual equation $A_{i} e_{i}=r_{i}$ in $\mathcal{V}_{i}$ approximately

$$
\hat{e}_{i}=R_{i} r_{i}
$$


Subspace corrections $\hat{e}_{i}$ are assembled to yield a correction in the space $\mathcal{V}$, thereby giving rise to the so-called method of subspace corrections. There are two basic ways to assemble subspace corrections.

Parallel Subspace Correction (PSC). This method performs the correction on each subspace in parallel. In operator form, it reads

$$
u^{k+1}=u^{k}+B\left(f-A u^{k}\right),
$$

where

$$
B=\sum_{i=0}^{J} I_{i} R_{i} I_{i}^{t}
$$

The subspace correction is $\hat{e}_{i}=I_{i} R_{i} I_{i}^{t}\left(f-A u^{k}\right)$, and the correction in $\mathcal{V}$ is $\hat{e}=\sum_{i=0}^{J} \hat{e}_{i}$. The error equation reads

$$
u-u^{k+1}=\left[I-\left(\sum_{i=0}^{J} I_{i} R_{i} I_{i}^{t}\right) A\right]\left(u-u^{k}\right)=\left(I-\sum_{i=0}^{J} T_{i}\right)\left(u-u^{k}\right) ;
$$

Successive Subspace Correction (SSC). This method performs the correction in a successive way. In operator form, it reads

$$
v^{0}=u^{k}, \quad v^{i+1}=v^{i}+I_{i} R_{i} I_{i}^{t}\left(f-A v^{i}\right), i=0, \ldots, J, \quad u^{k+1}=v^{J+1},
$$

and the corresponding error equation is

$$
u-u^{k+1}=\left[\prod_{i=0}^{J}\left(I-I_{i} R_{i} I_{i}^{t} A\right)\right]\left(u-u^{k}\right)=\left[\prod_{i=0}^{J}\left(I-T_{i}\right)\right]\left(u-u^{k}\right) ;
$$

in the notation $\prod_{i=0}^{J} a_{i}$, we assume there is a built-in ordering from $i=0$ to $J$, i.e., $\prod_{i=0}^{J} a_{i}=$ $a_{0} a_{1} \ldots a_{J}$. Therefore, PSC is an additive method whereas SSC is a multiplicative method.

As a trivial example, we consider the space decomposition $\mathbb{R}^{J}=\sum_{i=1}^{J} \operatorname{span}\left\{e_{i}\right\}$. In this case, if we use exact (one dimensional) subspace solvers, the resulting SSC is just the Gauss-Seidel method and the PSC is just the Jacobi method. More complicated and effective examples, including multigrid methods and multilevel preconditioners, will be discussed later on.

2.3. Sharp Convergence Identities. The analysis of parallel subspace correction methods relies on the following identity which is well known in the literature [59, 61, 28, 64].

THEOREM 2.1 (Identity for PSC). If $R_{i}$ is SPD on $\mathcal{V}_{i}$ for $i=0, \ldots, J$, then $B$ defined by (2.3) is also SPD on $\mathcal{V}$. Furthermore

$$
\left(B^{-1} v, v\right)=\inf _{\sum_{i=0}^{J} v_{i}=v} \sum_{i=0}^{J}\left(R_{i}^{-1} v_{i}, v_{i}\right) .
$$

On the other hand, the analysis of Successive subspace correction methods hinges on an identity of Xu and Zikatanov [64] to be described below. First we assume that each subspace smoother $R_{i}$ induces a convergent iteration, i.e. the error operator $I-T_{i}$ is a contraction. 
(T) Contraction of Subspace Error Operator: There exists $\rho<1$ such that

$$
\left\|I-T_{i}\right\|_{A_{i}} \leq \rho \text { for all } i=0,1, \cdots, J .
$$

We associate with $T_{i}$ the adjoint operator $T_{i}^{*}$ with respect to the inner product $(\cdot, \cdot)_{A}$. To deal with general, possibly non-symmetric smoothers $R_{i}$, we introduce the symmetrization of $T_{i}$

$$
\bar{T}_{i}=T_{i}+T_{i}^{*}-T_{i}^{*} T_{i}, \text { for } i=0, \cdots, J .
$$

We use a simplified version of XZ identity given by Cho, Xu, and Zikatanov [23]; see also [20].

THEOREM 2.2 (Identity of SSC). If assumption (T) is valid, then the following identity holds

$$
\left\|\prod_{i=0}^{J}\left(I-T_{i}\right)\right\|_{A}^{2}=1-\frac{1}{K}
$$

where

$$
K=\sup _{\|v\|_{A}=1} \inf _{\sum_{i=0}^{J} v_{i}=v} \sum_{k=0}^{J}\left(\bar{T}_{i}^{-1}\left(v_{i}+T_{i}^{*} w_{i}\right), v_{i}+T_{i}^{*} w_{i}\right)_{A},
$$

with $w_{i}=\sum_{j>i} v_{j}$.

When we choose exact local solvers, i.e., $R_{i}=A_{i}^{-1}$ and consequently $T_{i}=P_{i}$ for $i=0, \cdots, J,(\mathbf{T})$ holds with $\rho=0$. Therefore we have a more concise formulation for such choice [64].

COROLLARY 2.3 (Identity of SSC with exact solver). One has the following identity

$$
\left\|\prod_{i=0}^{J}\left(I-P_{i}\right)\right\|_{A}^{2}=1-\frac{1}{1+c_{0}}
$$

where

$$
c_{0}=\sup _{\|v\|_{A}=1} \inf _{\sum_{i=0}^{J} v_{i}=v} \sum_{i=0}^{J}\left\|P_{i} \sum_{j=i+1}^{N} v_{j}\right\|_{A}^{2} .
$$

2.4. Convergence Analysis. We now present a convergence analysis based on three assumptions: (T) on $T_{i}$ and the two ones below on the space decomposition. The analysis here is adapted from Xu [61] and simplified by using the XZ identity.

(A1) Stable Decomposition: For any $v \in \mathcal{V}$, there exists a decomposition $v=\sum_{i=0}^{J} v_{i}, v_{i} \in$ $\mathcal{V}_{i}, i=0, \ldots, J$ such that

$$
\sum_{i=0}^{J}\left\|v_{i}\right\|_{A}^{2} \leq K_{1}\|v\|_{A}^{2}
$$

(A2) Strengthened Cauchy Schwarz (SCS) Inequality: For any $u_{i}, v_{i} \in \mathcal{V}_{i}, i=0, \cdots, J$

$$
\left|\sum_{i=0}^{J} \sum_{j=i+1}^{J}\left(u_{i}, v_{j}\right)_{A}\right| \leq K_{2}\left(\sum_{i=0}^{J}\left\|u_{i}\right\|_{A}^{2}\right)^{1 / 2}\left(\sum_{i=0}^{J}\left\|v_{i}\right\|_{A}^{2}\right)^{1 / 2} \text {. }
$$


THEOREM 2.4 (Multilevel preconditioning). Let $\mathcal{V}=\sum_{i=0}^{J} \mathcal{V}_{i}$ be a space decomposition satisfying assumptions (A1) and (A2), and let $R_{i}$ be SPDs for $i=0, \cdots, J$ such that

$$
K_{4}^{-1}\left\|u_{i}\right\|_{A}^{2} \leq\left(R_{i}^{-1} u_{i}, u_{i}\right) \leq K_{3}\left\|u_{i}\right\|_{A}^{2} .
$$

Then $B$ defined by (2.3) is SPD and

$$
\kappa(B A) \leq\left(1+2 K_{2}\right) K_{1} K_{3} K_{4} .
$$

Proof. Let $v=\sum_{i=0}^{J} v_{i}$ be a decomposition satisfying (2.10). It follows from the identity (2.5), and the definitions (2.10) of $K_{1}$ and (2.12) of $K_{3}$, that

$$
\left(B^{-1} v, v\right) \leq \sum_{i=0}^{J}\left(R_{i}^{-1} v_{i}, v_{i}\right) \leq K_{3} \sum_{i=0}^{J}\left\|v_{i}\right\|_{A}^{2} \leq K_{1} K_{3}\|v\|_{A}^{2}=K_{1} K_{3}(A v, v),
$$

which implies

$$
\lambda_{\min }(B A) \geq\left(K_{1} K_{3}\right)^{-1}
$$

For any decomposition $v=\sum_{i=0}^{J} v_{i}$, in view of (2.11) and (2.12), we have

$$
\begin{aligned}
(A v, v) & \leq \sum_{i=0}^{J}\left(v_{i}, v_{i}\right)_{A}+2\left|\sum_{i=0}^{J} \sum_{j=i+1}^{J}\left(v_{i}, v_{j}\right)_{A}\right| \\
& \leq\left(1+2 K_{2}\right) \sum_{i=0}^{J}\left\|v_{i}\right\|_{A}^{2} \leq\left(1+2 K_{2}\right) K_{4} \sum_{i=0}^{J}\left(R_{i}^{-1} v_{i}, v_{i}\right) .
\end{aligned}
$$

Taking the infimum and using again (2.5), we get

$$
(A v, v) \leq\left(1+2 K_{2}\right) K_{4} \inf _{\sum_{k=0}^{J} v_{i}=v} \sum_{i=0}^{J}\left(R_{i}^{-1} v_{i}, v_{i}\right)=\left(1+2 K_{2}\right) K_{4}\left(B^{-1} v, v\right),
$$

which implies

$$
\lambda_{\max }(B A) \leq\left(1+2 K_{2}\right) K_{4}
$$

The estimate (2.13) then follows from (2.14) and (2.15). $\square$

LEMMA 2.5 (Estimate of eigenvalues of $\bar{T}_{i}^{-1}$ ). If $(\mathbf{T})$ holds, then $\bar{T}_{i}$ is non-singular and

$$
1 \leq \lambda_{\min }\left(\bar{T}_{i}^{-1}\right) \leq \lambda_{\max }\left(\bar{T}_{i}^{-1}\right) \leq \frac{1}{1-\rho^{2}} .
$$

Proof. The estimates follow easily by (2.6) and the definition of $\bar{T}_{i}$, which satisfies

$$
I-\bar{T}_{i}=\left(I-T_{i}^{*}\right)\left(I-T_{i}\right)=\left(I-T_{i}\right)^{*}\left(I-T_{i}\right) .
$$

We omit the details. $\square$

THEOREM 2.6 (Convergence of SSC). Let $\mathcal{V}=\sum_{i=0}^{J} \mathcal{V}_{i}$ be a space decomposition satisfying assumptions (A1) and (A2), and let the subspace smoothers $T_{i}$ satisfy $(\mathbf{T})$. We then have

$$
\left\|\prod_{i=0}^{J}\left(I-T_{i}\right)\right\|_{A}^{2} \leq 1-\frac{1-\rho^{2}}{2 K_{1}\left(1+(1+\rho)^{2} K_{2}^{2}\right)}
$$


Proof. We shall give an upper bound of the constant $K$ in Theorem 2.2 by choosing a stable decomposition $v=\sum_{i} v_{i}$ satisfying (2.10). By the inequality $2 a b \leq a^{2}+b^{2}$, we have

$$
2\left(\bar{T}_{i}^{-1} v_{i}, T_{i}^{*} w_{i}\right)_{A} \leq\left(\bar{T}_{i}^{-1} v_{i}, v_{i}\right)_{A}+\left(\bar{T}_{i}^{-1} T_{i}^{*} w_{i}, T_{i}^{*} w_{i}\right)_{A},
$$

where $w_{j}=\sum_{j>1} v_{j}$. Therefore we only need to estimate the two terms on the right hand side of (2.18).

Using the eigenvalue estimate of $\bar{T}_{i}^{-1}$ in Lemma 2.5, together with (2.10), we arrive at

$$
\sum_{i=0}^{J}\left(\bar{T}_{i}^{-1} v_{i}, v_{i}\right)_{A} \leq \sum_{i=0}^{J} \lambda_{\max }\left(\bar{T}_{i}^{-1}\right)\left\|v_{i}\right\|_{A}^{2} \leq \frac{K_{1}}{1-\rho^{2}}\|v\|_{A}^{2} .
$$

To estimate the second term in (2.18), we use the SCS estimate (2.11) to get

$$
\begin{aligned}
\sum_{i=0}^{J}\left(\bar{T}_{i}^{-1} T_{i}^{*} w_{i}, T_{i}^{*} w_{i}\right)_{A} & =\sum_{i=0}^{J} \sum_{j=i+1}^{J}\left(T_{i} \bar{T}_{i}^{-1} T_{i}^{*} w_{i}, v_{j}\right)_{A} \\
& \leq K_{2}\left(\sum_{i=0}^{J}\left\|T_{i} \bar{T}_{i}^{-1} T_{i}^{*} w_{i}\right\|_{A}^{2}\right)^{1 / 2}\left(\sum_{i=0}^{J}\left\|v_{i}\right\|_{A}^{2}\right)^{1 / 2}
\end{aligned}
$$

Since $\left\|T_{i}\right\|_{A_{i}}=\left\|T_{i}^{*}\right\|_{A_{i}} \leq 1+\rho$ and $\left\|\bar{T}_{i}^{-1}\right\|_{A_{i}}=\lambda_{\max }\left(\bar{T}_{i}^{-1}\right) \leq \frac{1}{1-\rho^{2}}$, we deduce

$$
\left\|T_{i} \bar{T}_{i}^{-1} T_{i}^{*} w_{i}\right\|_{A} \leq\left\|T_{i}\right\|_{A_{i}}\left\|\bar{T}_{i}^{-1}\right\|_{A_{i}}\left\|T_{i}^{*}\right\|_{A_{i}}\left\|w_{i}\right\|_{A} \leq \frac{1+\rho}{1-\rho}\left\|w_{i}\right\|_{A} .
$$

Now using the SCS estimate (2.11) again, we get

$$
\sum_{i=0}^{J}\left(w_{i}, w_{i}\right)_{A}=\sum_{i=0}^{J} \sum_{j=i+1}^{J}\left(w_{i}, v_{j}\right)_{A} \leq K_{2}\left(\sum_{i=0}^{J}\left\|w_{i}\right\|_{A}^{2}\right)^{1 / 2}\left(\sum_{i=0}^{J}\left\|v_{i}\right\|_{A}^{2}\right)^{1 / 2},
$$

which leads to

$$
\sum_{i=0}^{J}\left\|w_{i}\right\|_{A}^{2} \leq K_{2}^{2} \sum_{i=0}^{J}\left\|v_{i}\right\|_{A}^{2}
$$

Consequently,

$$
\sum_{i=0}^{J}\left(\bar{T}_{i}^{-1} T_{i}^{*} w_{i}, T_{i}^{*} w_{i}\right)_{A} \leq K_{2}^{2} \frac{1+\rho}{1-\rho} \sum_{i=0}^{J}\left\|v_{i}\right\|_{A}^{2} \leq K_{1} K_{2}^{2} \frac{1+\rho}{1-\rho}\|v\|_{A}^{2} .
$$

Inserting (2.18), (2.19), and (2.18) into (2.8), we get the upper bound of $K \leq 2 K_{1}(1+(1+$ $\left.\rho)^{2} K^{2}\right) /\left(1-\rho^{2}\right)$. Finally, the desired contraction estimate follows from Theorem 2.2.

When we use exact local solvers $R_{i}=A_{i}^{-1}$, we have a simpler proof and sharper estimate.

COROLLARY 2.7. Let the space decomposition satisfy (A1) and (A2), and let $R_{i}=A_{i}^{-1}$ for all $i$. Then

$$
\left\|\prod_{i=0}^{J}\left(I-P_{i}\right)\right\|_{A}^{2} \leq 1-\frac{1}{1+K_{1} K_{2}^{2}}
$$


Proof. We apply (2.11) with $u_{i}=P_{i} \sum_{j=i+1}^{J} v_{j}$ to obtain

$$
\begin{aligned}
\sum_{i=0}^{J}\left\|u_{i}\right\|_{A}^{2} & =\sum_{i=0}^{J}\left(u_{i}, P_{i} \sum_{j=i+1}^{J} v_{j}\right)_{A}=\sum_{i=0}^{J} \sum_{j=i+1}^{J}\left(u_{i}, v_{j}\right)_{A} \\
& \leq K_{2}\left(\sum_{i=0}^{J}\left\|u_{i}\right\|_{A}^{2}\right)^{1 / 2}\left(\sum_{i=0}^{J}\left\|v_{i}\right\|_{A}^{2}\right)^{1 / 2}
\end{aligned}
$$

Consequently, if $v=\sum_{k=0}^{J} v_{k}$ is a stable decomposition satisfying (2.10), we get

$$
\sum_{i=0}^{J}\left\|P_{i} \sum_{j=i+1}^{J} v_{j}\right\|_{A}^{2}=\sum_{i=0}^{J}\left\|u_{i}\right\|_{A}^{2} \leq K_{2}^{2} \sum_{i=0}^{J}\left\|v_{i}\right\|_{A}^{2} \leq K_{1} K_{2}^{2}\|v\|_{A}^{2},
$$

which implies $c_{0} \leq K_{2}^{2} K_{1}$. The desired result then follows from Corollary 2.3. $\square$

3. Bisection Methods. We now discuss bisection methods for simplicial grids for $d \geq$ 2 , following [53, 42], and present a novel decomposition of conforming meshes obtained by bisection. We do not discuss the alternative refinement method, called regular refinement, which divides one simplex into $2^{d}$ children; see [7,24] for $d=2$ and $[11,1]$ for $d=3$.

3.1. Bisection Rules. Given a simplex $\tau$, we assign one of its edges as the refinement edge of $\tau$. Starting from an initial triangulation $\mathcal{T}_{0}$, a bisection method consists of the following rules:

R1. Assign refinement edges for each element $\tau \in \mathcal{T}_{0}$ (initial labeling);

R2. Divide a simplex into two simplices by joining the midpoint of its refinement edge with its vertices other than those in the refinement edge (bisection);

R3. Assign refinement edges to the two children of a bisected simplex (labeling).

There are several bisection methods proposed for $d \geq 3$ [8, 32, 33, 46, 3, 54], which generalize the newest vertex bisection [37] and longest edge bisection [48] for $d=2$. We now give a mathematical description based on Kossaczky [32], Traxler [55], and Stevenson [54]. For each simplex $\tau$, rules R1-3 associate a unique refinement edge $e$. The pair $(\tau, e)$ is called labeled simplex, and $(\mathcal{T}, \mathcal{L}):=\{(\tau, e): \tau \in \mathcal{T}\}$ is called labeled triangulation. A $d$-simplex $\tau$ is a set of $d+1$ ordered vertices $\left\{x_{i}\right\}_{i=0}^{d}$ and type $t$ :

$$
\tau=\left\{x_{0}, x_{1}, \cdots, x_{d}\right\}, \quad t \in\{0,1, \cdots, d\} .
$$

We let $e=\overline{x_{0} x_{d}}$ be the refinement edge, and let $\bar{x}=\frac{1}{2}\left(x_{0}+x_{d}\right)$ be the midpoint of $e$. The two children $\tau_{1}, \tau_{2}$ of $\tau$ are the simplices obtained by joining $\bar{x}$ with the vertices of $\tau$ other than $x_{0}, x_{d}$. Ordering the vertices of the children, or equivalently labeling them, is a crucial process that includes R2-3. We consider the following rule R3:

$$
\begin{aligned}
\tau_{1} & :=\{x_{0}, \bar{x}, \underbrace{x_{1}, \ldots, x_{t}}_{\rightarrow}, \underbrace{x_{t+1}, \ldots, x_{d-1}}_{\rightarrow}\}_{(t+1) \bmod d}, \\
\tau_{2} & :=\{x_{d}, \bar{x}, \underbrace{x_{1}, \ldots, x_{t}}_{\rightarrow}, \underbrace{x_{d-1}, \ldots, x_{t+1}}_{\leftarrow}\}_{(t+1) \bmod d},
\end{aligned}
$$

with the convention that arrows point in the direction of increasing indices and $\left\{x_{1}, \ldots, x_{0}\right\}=$ $\emptyset,\left\{x_{d}, \ldots, x_{d-1}\right\}=\emptyset$. For $d=2$ rule R3 does not depend on the element type and we get 
for $\tau=\left\{x_{0}, x_{1}, x_{2}\right\}$ the two children $\tau_{1}=\left\{x_{0}, \bar{x}, x_{1}\right\}, \tau_{2}=\left\{x_{2}, \bar{x}, x_{1}\right\}$. Moreover, the refinement edge of the two children is opposite to the new vertex $\bar{x}$, whence this procedure coincides with the newest vertex bisection method. We refer to the survey [42, Section 4] for a discussion for $d \geq 2$, and stress that once rule R1 is settled, then the subsequent labeled grids are uniquely defined.

For a labeled triangulation $(\mathcal{T}, \mathcal{L})$, and $\tau \in \mathcal{T}$, a bisection $b_{\tau}:\{(\tau, e)\} \rightarrow\left\{\left(\tau_{1}, e_{1}\right),\left(\tau_{2}, e_{2}\right)\right\}$ is a map that encodes the above procedure. We next define the formal addition as follows:

$$
\mathcal{T}+b_{\tau}:=(\mathcal{T}, \mathcal{L}) \backslash\{(\tau, e)\} \cup\left\{\left(\tau_{1}, e_{1}\right),\left(\tau_{2}, e_{2}\right)\right\}
$$

For an ordered sequence of bisections $\mathcal{B}=\left\{b_{\tau_{1}}, b_{\tau_{2}}, \cdots, b_{\tau_{N}}\right\}$, we define

$$
\mathcal{T}+\mathcal{B}:=\left(\left(\mathcal{T}+b_{\tau_{1}}\right)+b_{\tau_{2}}\right)+\cdots+b_{\tau_{N}}
$$

whenever the addition is well defined (i.e. $\tau_{i}$ should exists in the previous labeled triangulation). These additions are a convenient mathematical description of bisection on triangulations.

Given an initial grid $\mathcal{T}_{0}$ of $\Omega$ and rules R1-3, we define the sets

$$
\begin{aligned}
& \mathbb{G}\left(\mathcal{T}_{0}\right)=\left\{\mathcal{T}: \text { there exists a bisection sequence } \mathcal{B} \text { such that } \mathcal{T}=\mathcal{T}_{0}+\mathcal{B}\right\} \\
& \mathbb{T}\left(\mathcal{T}_{0}\right)=\left\{\mathcal{T} \in \mathbb{G}\left(\mathcal{T}_{0}\right): \mathcal{T} \text { is conforming }\right\} .
\end{aligned}
$$

Therefore $\mathbb{G}\left(\mathcal{T}_{0}\right)$ contains all (possibly nonconforming) grids obtained from $\mathcal{T}_{0}$ using the bisection method, which are uniquely defined once the rules R1-3 have been set, whereas $\mathbb{T}\left(\mathcal{T}_{0}\right)$ is the subset of conforming grids.

It is essential for the discussion to define the sequence of uniformly refined meshes $\left\{\overline{\mathcal{T}}_{k}\right\}_{k=0}^{\infty}$ by:

$$
\overline{\mathcal{T}}_{k}:=\overline{\mathcal{T}}_{k-1}+\left\{b_{\tau}: \tau \in \overline{\mathcal{T}}_{k-1}\right\}, \quad \text { for } k \geq 1
$$

with $\overline{\mathcal{T}}_{0}:=\mathcal{T}_{0}$. This means that $\overline{\mathcal{T}}_{k}$ is obtained by bisecting all elements in $\overline{\mathcal{T}}_{k-1}$ only once. Note that $\overline{\mathcal{T}}_{k} \in \mathbb{G}\left(\mathcal{T}_{0}\right)$ but not necessarily in $\mathbb{T}\left(\mathcal{T}_{0}\right)$.

We thus consider bisection methods which satisfy the following two assumptions:

(B1) Shape Regularity: $\mathbb{G}\left(\mathcal{T}_{0}\right)$ is shape regular.

(B2) Conformity of Uniform Refinement: $\overline{\mathcal{T}}_{k} \in \mathbb{T}\left(\mathcal{T}_{0}\right)$, i.e. $\overline{\mathcal{T}}_{k}$ is conforming, for all $k \geq 0$.

With the specific rule $(3.1)$, due to $[32,55,54]$, we see that the type $t$ increases by 1 and the vertex ordering changes with $t$, which in turn implies that after $d$ recurrent bisections of a simplex $\tau$ all its edges are bisected. This leads to a fixed number of similarity classes of elements, depending only on $\mathcal{T}_{0}$, and thus B1 holds for any $d$. We refer to [42] for a thorough discussion.

We recall that for $d=2$, rule (3.1) reduces to the newest vertex bisection, in which case Sewell [51] showed that all the descendants of a triangle in $\mathcal{T}_{0}$ fall into four similarity classes and hence (B1) holds. Note that (B2) may not hold for an arbitrary rule R1, namely the refinement edge for elements in the initial triangulation cannot be selected freely. Mitchell [37] came up with a rule R1 for which (B2) holds. He proved the existence of such initial labeling scheme (so-called compatible initial labeling), and Biedl, Bose, Demaine, and Lubiw [9] gave an optimal $\mathcal{O}(N)$ algorithm to find a compatible initial labeling for a triangulation with $N$ elements. In summary, for $d=2$, newest vertex bisection with compatible initial labeling is a bisection method which satisfies (B1) and (B2). 
Enforcing (B2) for $d>2$ requires also a labeling of the initial mesh $\mathcal{T}_{0}$, for which there is no constructive procedure. The algorithms proposed by Kossaczký [32] for $d=3$ and Stevenson [54] for $d \geq 3$ enforce such initial labeling upon further refining every element of the initial triangulation, which deteriorates the shape regularity. Although (B2) imposes a severe restriction on the initial labeling, we emphasize that it is also used to prove the optimal cardinality of adaptive finite element methods [53, 18, 42]. Finding conditions weaker than (B2) is a challenging open problem.

3.2. Compatible Bisections. We denote by $\mathcal{N}(\mathcal{T})$ the set of vertices of the triangulation $\mathcal{T}$ and by $\mathcal{E}(\mathcal{T})$ the set of all edges of $\mathcal{T}$. By convention, all simplices are closed sets. For a vertex $x \in \mathcal{N}(\mathcal{T})$ or an edge $e \in \mathcal{E}(\mathcal{T})$, we define the first ring (or the star) of $x$ or $e$ to be

$$
\mathcal{R}_{x}=\{\tau \in \mathcal{T} \mid x \in \tau\}, \quad \mathcal{R}_{e}=\{\tau \in \mathcal{T} \mid e \subset \tau\},
$$

and the local patch of $x$ or $e$ as $\omega_{x}=\cup_{\tau \in \mathcal{R}_{x}} \tau$, and $\omega_{e}=\cup_{\tau \in \mathcal{R}_{e}} \tau$. Note that $\omega_{x}$ and $\omega_{e}$ are subsets of $\Omega$, while $\mathcal{R}_{x}$ and $\mathcal{R}_{e}$ are subsets of $\mathcal{T}$ which can be thought of as triangulations of $\omega_{x}$ and $\omega_{e}$, respectively. We indicate with $\# S$ the cardinality of a set $S$.

Given a labeled triangulation $(\mathcal{T}, \mathcal{L})$, an edge $e \in \mathcal{E}(\mathcal{T})$ is called a compatible edge if $e$ is the refinement edge of $\tau$ for all $\tau \in \mathcal{R}_{e}$. For a compatible edge $e$, the ring $\mathcal{R}_{e}$ is called a compatible ring, and the patch $\omega_{e}$ is called a compatible patch. Let $x$ be the midpoint of a compatible edge $e$ and $\mathcal{R}_{x}$ be the ring of $x$ in $\mathcal{T}+\left\{b_{\tau}: \tau \in \mathcal{R}_{e}\right\}$. A compatible bisection is a mapping $b_{e}: \mathcal{R}_{e} \rightarrow \mathcal{R}_{x}$. We then define the addition

$$
\mathcal{T}+b_{e}:=\mathcal{T}+\left\{b_{\tau}: \tau \in \mathcal{R}_{e}\right\}=\mathcal{T} \backslash \mathcal{R}_{e} \cup \mathcal{R}_{x} .
$$

Note that if $\mathcal{T}$ is conforming, then $\mathcal{T}+b_{e}$ is conforming for a compatible bisection $b_{e}$, whence compatible bisections preserve conformity of triangulations and are thus a fundamental concept both in theory and practice. For a compatible bisection sequence $\mathcal{B}=\left\{b_{i}\right\}_{i=0}^{J}$, the addition $\mathcal{T}_{0}+\mathcal{B}$ is defined recursively $\mathcal{T}_{i}=\mathcal{T}_{i-1}+b_{i}$ for $1 \leq i \leq J$.
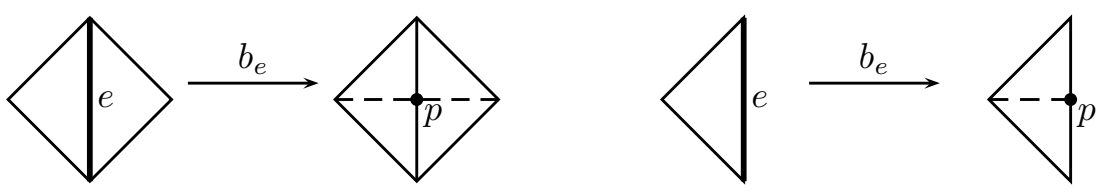

FIG. 3.1. Two compatible bisections for $d=2$. Left: interior edge; right: boundary edge. The edge with boldface is the compatible refinement edge, and the dash-line represents the bisection.

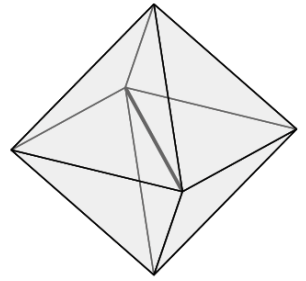

(a) A compatible patch $\omega_{e}$ with common edge $e$

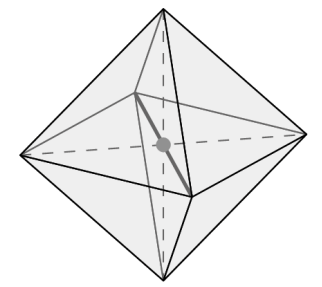

(b) Patch $\omega_{p}$ after a compatible bisection $b_{p}$

FIG. 3.2. A compatible bisection for $d=3$ : the edge e (in bold) is the refinement edge of all elements in the patch $\omega_{e}$. Connecting the midpoint $p$ of e to the other vertices bisects each element of the compatible ring $\mathcal{R}_{e}$ and keeps the mesh conforming without spreading refinement outside $\omega_{e}$. This is an atomic operation. 
In two dimensions, a compatible bisection $b_{e}$ has only two possible configurations; see Fig. 3.1. The first one corresponds to bisecting an interior compatible edge, in which case the patch $\omega_{e}$ is a quadrilateral. The second case corresponds to bisecting a boundary edge, which is always compatible, and $\omega_{e}$ is a triangle. In three dimensions, the configuration of compatible bisections depends on the initial labeling; see Fig. 3.2 for a simple case.

The bisection of paired triangles was first introduced by Mitchell for dimension $d=$ 2 [37, 38]. The idea was generalized by Kossaczký [32] to $d=3$, and Maubach [33] and Stevenson [54] to $d \geq 2$. In the aforementioned references, efficient recursive completion procedures of bisection methods are introduced based on compatible bisections. We use them to characterize the conforming mesh obtained by bisection methods.

3.3. Decomposition of Bisection Grids. We now present a decomposition of meshes in $\mathbb{T}\left(\mathcal{T}_{0}\right)$ using compatible bisections, which will be instrumental later.

THEOREM 3.1 (Decomposition of bisection grids). Let $\mathcal{T}_{0}$ be a conforming triangulation. Suppose the bisection method satisfies assumption (B2), i.e., for all $k \geq 0$ all uniform refinements $\overline{\mathcal{T}}_{k}$ of $\mathcal{T}_{0}$ are conforming. Then for any $\mathcal{T} \in \mathbb{T}\left(\mathcal{T}_{0}\right)$, there exists a compatible bisection sequence $\mathcal{B}=\left\{b_{1}, b_{2}, \cdots, b_{N}\right\}$ with $N=\# \mathcal{N}(\mathcal{T})-\# \mathcal{N}\left(\mathcal{T}_{0}\right)$ such that

$$
\mathcal{T}=\mathcal{T}_{0}+\mathcal{B} .
$$

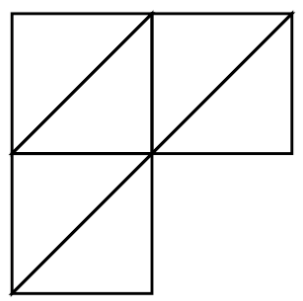

(a) Initial grid $\mathcal{T}_{0}$

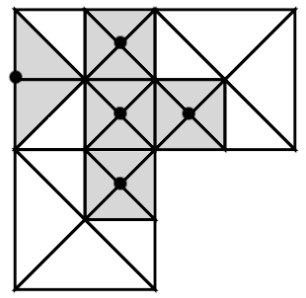

(d) $\mathcal{T}_{13}$

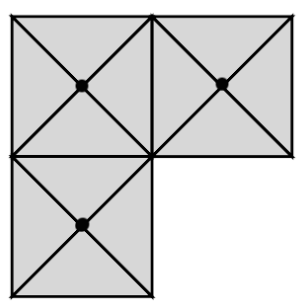

(b) $\mathcal{T}_{3}$

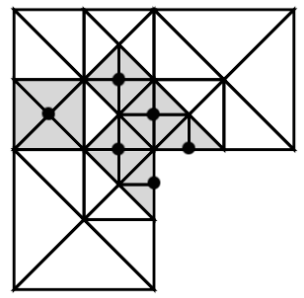

(e) $\mathcal{T}_{19}$

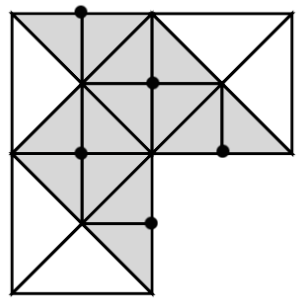

(c) $\mathcal{T}_{8}$

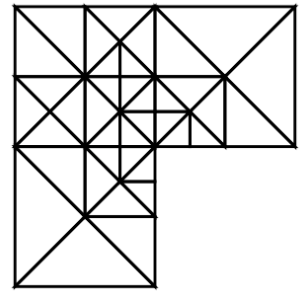

(f) Fine grid $\mathcal{T}=\mathcal{T}_{19}$

FIG. 3.3. Decomposition of a bisection grid for $d=2$ : Each frame displays a mesh $\mathcal{T}_{i+k}=\mathcal{T}_{i}+$ $\left\{b_{i+1}, \cdots, b_{i+k}\right\}$ obtained from $\mathcal{T}_{i}$ by a sequence of compatible bisections $\left\{b_{j}\right\}_{j=i+1}^{i+k}$ using the longest edge. The order of bisections is irrelevant within each frame, but matters otherwise.

We prove this result later in this section. We now use the example in Figure 3.3 to illustrate the decomposition of a bisection grid. In Figure 3.3 (a), we display the initial triangulation $\mathcal{T}_{0}$ which uses the longest edge as the refinement edge for each triangle. We display the fine grid $\mathcal{T} \in \mathbb{T}\left(\mathcal{T}_{0}\right)$ in Figure 3.3 (f). In Figure 3.3 (b)-(e), we give several intermediate triangulations during the refinement process: each triangulation is obtained by performing 
several compatible bisections on the previous one. Each compatible patch is indicated by a gray region and the new vertices introduced by bisections are marked by black dots.

To prove Theorem 3.1, we introduce the generation of elements and vertices. The generation of each element in the initial grid $\mathcal{T}_{0}$ is defined to be 0 , and the generation of an element $\tau \in \mathbb{G}\left(\mathcal{T}_{0}\right)$ is 1 plus that of the father. Therefore, $g_{\tau}$ coincides with the number of bisections needed to create $\tau$ from $\mathcal{T}_{0}$, and the uniformly refined mesh $\overline{\mathcal{T}}_{k}$ can be characterized as the triangulation in $\mathbb{G}\left(\mathcal{T}_{0}\right)$ with all its elements of the same generation $k$. Vice versa, an element $\tau$ with generation $k$ can exist in $\overline{\mathcal{T}}_{j}$ only for $j=k$.

Let $\mathbb{N}\left(\mathcal{T}_{0}\right)=\cup\left\{\mathcal{N}(\mathcal{T}): \mathcal{T} \in \mathbb{G}\left(\mathcal{T}_{0}\right)\right\}$ denote the set of all possible vertices. For any vertex $p \in \mathbb{N}\left(\mathcal{T}_{0}\right)$, the generation $g_{p}$ of $p$ is defined as the minimal integer $k$ such that $p \in \mathcal{N}\left(\overline{\mathcal{T}}_{k}\right)$.

The following properties about the generation of elements or vertices for uniformly refined meshes $\overline{\mathcal{T}}_{k}$ are a consequence of the definition above:

$$
\begin{array}{r}
\tau \in \overline{\mathcal{T}}_{k} \text { if and only if } g_{\tau}=k ; \\
p \in \mathcal{N}\left(\overline{\mathcal{T}}_{k}\right) \text { if and only if } g_{p} \leq k ; \\
\text { for } \tau \in \overline{\mathcal{T}}_{k}, \max _{q \in \mathcal{N}(\tau)} g_{q}=k=g_{\tau},
\end{array}
$$

where $\mathcal{N}(\tau)$ is the set of vertices of $\tau \in \overline{\mathcal{T}}_{k}$.

LEMMA 3.2 (Maximal generation). Let $\mathcal{T}_{0}$ be a conforming triangulation. Let the bisection method satisfy assumption (B2). For any $\mathcal{T} \in \mathbb{T}\left(\mathcal{T}_{0}\right)$, let $p \in \mathcal{N}(\mathcal{T})$ be a vertex with maximal generation in the sense that $g_{p}=\max _{q \in \mathcal{N}(\mathcal{T})} g_{q}$. Then, all elements in $\mathcal{R}_{p}$ have the same generation $g_{p}$, namely,

$$
\begin{aligned}
g_{\tau} & =g_{p} \quad \text { for all } \tau \in \mathcal{R}_{p}, \\
\mathcal{R}_{p} & =\overline{\mathcal{R}}_{k, p},
\end{aligned}
$$

where $k=g_{p}$ and $\overline{\mathcal{R}}_{k, p}$ is the first ring of $p$ in the uniformly refined mesh $\overline{\mathcal{T}}_{k}$.

Proof. We prove (3.6) by showing $g_{p} \leq g_{\tau}$ and $g_{\tau} \leq g_{p}$. Since $\mathcal{T}$ is conforming, $p$ is a vertex of each element $\tau \in \mathcal{R}_{p}$. This implies that $p \in \mathcal{\mathcal { N }}\left(\overline{\mathcal{T}}_{g_{\tau}}\right)$ and thus $g_{\tau} \geq g_{p}$ by (3.4). On the other hand, from (3.5), we have

$$
g_{\tau}=\max _{q \in \mathcal{N}(\tau)} g_{q} \leq \max _{q \in \mathcal{N}(\mathcal{T})} g_{q}=g_{p}, \quad \text { for all } \tau \in \mathcal{R}_{p}
$$

Now we prove (3.7). By (3.3), $\overline{\mathcal{R}}_{k, p}$ is made of all elements with generation $k$ containing $p$. By (3.6), we conclude $\mathcal{R}_{p} \subseteq \overline{\mathcal{R}}_{k, p}$. The fact that $p$ cannot belong to the domain $\Omega \backslash \omega_{p}$, because of the topology of $\omega_{p}$, implies $\overline{\mathcal{R}}_{k, p} \backslash \mathcal{R}_{p}=\varnothing$. This proves (3.7). $\square$

Now we are in the position to prove Theorem 3.1.

Proof of Theorem 3.1. We prove the result by induction on $N=\# \mathcal{N}(\mathcal{T})-\# \mathcal{N}\left(\mathcal{T}_{0}\right)$. Nothing needs to be proved for $N=0$. Assume that (3.2) holds for $N$.

Let $\mathcal{T} \in \mathbb{T}\left(\mathcal{T}_{0}\right)$ with $\# \mathcal{N}(\mathcal{T})-\# \mathcal{N}\left(\mathcal{T}_{0}\right)=N+1$. Let $p \in \mathcal{N}(\mathcal{T})$ be a vertex with maximal generation, i.e., $g_{p}=\max _{q \in \mathcal{N}(\mathcal{T})} g_{q}$. Then by Lemma 3.2, we know that $\mathcal{R}_{p}=$ $\overline{\mathcal{R}}_{k, p}$ for $k=g_{p}$. Now by assumption (B2), $\overline{\mathcal{R}}_{k, p}$ is created by a compatible bisection, say

$$
b_{e}: \overline{\mathcal{R}}_{e} \rightarrow \overline{\mathcal{R}}_{k, p}
$$

with $e \in \mathcal{E}\left(\overline{\mathcal{T}}_{k-1}\right)$. Since the compatible bisection giving rise to $p$ is unique within $\mathbb{G}\left(\mathcal{T}_{0}\right)$, it must thus be $b_{e}$. This means that if we undo the bisection operation, then we still have a conforming mesh $\mathcal{T}^{\prime}$, or equivalently $\mathcal{T}=\mathcal{T}^{\prime}+b_{e}$. We can now apply the induction assumption to $\mathcal{T}^{\prime} \in \mathbb{T}\left(\mathcal{T}_{0}\right)$ with $\# \mathcal{N}\left(\mathcal{T}^{\prime}\right)-\# \mathcal{N}\left(\mathcal{T}_{0}\right)=N$ to finish the proof. 
3.4. Generation of Compatible Bisections. For a compatible bisection $b_{i} \in \mathcal{B}$, we use the same subscript $i$ to denote related quantities such as:

- $e_{i}$ : the refinement edge;

- $p_{i}$ : the midpoint of $e_{i}$;

- $\widetilde{\omega}_{i}=\omega_{p_{i}} \cup \omega_{p_{l_{i}}} \cup \omega_{p_{p_{i}}}$;

- $\mathcal{T}_{i}=\mathcal{T}_{0}+\left\{b_{1}, \cdots, b_{i}\right\}$;
- $\omega_{i}$ : the patch of $p_{i}$ i.e. $\omega_{p_{i}}$;

- $p_{l_{i}}, p_{r_{i}}$ : two end points of $e_{i}$;

- $h_{i}$ : the local mesh size of $\omega_{i}$;

- $\mathcal{R}_{i}$ : the first ring of $p_{i}$ in $\mathcal{T}_{i}$.

We understand $h \in L^{\infty}(\Omega)$ as a piecewise constant mesh-size function, i.e., $h_{\tau}=$ $\operatorname{diam}(\tau)$ in each simplex $\tau \in \mathcal{T}$.

LEMma 3.3 (Compatibility and generation). If $b_{i} \in \mathcal{B}$ is a compatible bisection, then all elements of $\mathcal{R}_{i}$ have the same generation $g_{i}$.

Proof. Let $p_{i} \in \mathcal{N}\left(\mathcal{T}_{i}\right)$ be the vertex associated with $b_{i}$. Let $\overline{\mathcal{T}_{i}}$ be the coarsest uniformly refined mesh containing $p_{i}$, so $k=g_{p_{i}}$. In view of assumption (B2), $p_{i}$ arises from uniform refinement of $\overline{\mathcal{T}}_{k-1}$. Since the bisection giving rise to $p_{i}$ is unique within $\mathbb{F}\left(\mathcal{T}_{0}\right)$, we realize that all elements in $\mathcal{R}_{e_{i}}$ are bisected and have generation $k-1$ because they belong to $\overline{\mathcal{T}}_{k-1}$. This implies that all elements of $\mathcal{R}_{p_{i}}$ have generation $k$, as asserted. $\square$

This lemma enables us to introduce the concept of generation of compatible bisections. For a compatible bisection $b_{i}: \mathcal{R}_{e_{i}} \rightarrow \mathcal{R}_{p_{i}}$, we define $g_{i}=g(\tau), \tau \in \mathcal{R}_{p_{i}}$. Throughout this paper we always assume $h(\tau) \approx 1$ for $\tau \in \mathcal{T}_{0}$. We thus have the following important relation between generation and mesh size

$$
h_{i} \approx \gamma^{g_{i}}, \text { with } \gamma=\left(\frac{1}{2}\right)^{1 / d} \in(0,1) .
$$

Besides this relation, we give now two more important properties on the generation of compatible bisections. The first property says that different bisections with the same generation have weakly disjoint local patches.

LEMMA 3.4 (Nonoverlapping patches). Let $\mathcal{T}_{N} \in \mathbb{T}\left(\mathcal{T}_{0}\right)$ be $\mathcal{T}_{N}=\mathcal{T}_{0}+\mathcal{B}$, where $\mathcal{B}$ is a compatible bisection sequence $\mathcal{B}=\left\{b_{1}, \cdots, b_{N}\right\}$. For any $i \neq j$ and $g_{j}=g_{i}$, we have

$$
\stackrel{\circ}{\omega}_{i} \cap \stackrel{\circ}{\omega}_{j}=\varnothing .
$$

Proof. Since $g_{i}=g_{j}=g$, both bisection patches $\mathcal{R}_{i}$ and $\mathcal{R}_{j}$ belong to the uniformly refined mesh $\overline{\mathcal{T}}_{g}$. If (3.9) were not true, then there would exists $\tau \in \mathcal{R}_{i} \cap \mathcal{R}_{j} \subset \overline{\mathcal{T}}_{g}$ containing distinct refinement edges $e_{i}$ and $e_{j}$ because $i \neq j$. This contradicts rules R2 and R3 which assign a unique refinement edge to each element.

A simple consequence of (3.9) is that, for all $u \in L^{2}(\Omega)$ and $k \geq 1$,

$$
\begin{aligned}
& \sum_{g_{i}=k}\|u\|_{\omega_{i}}^{2} \leq\|u\|_{\Omega}^{2}, \\
& \sum_{g_{i}=k}\|u\|_{\tilde{\omega}_{i}}^{2} \lesssim\|u\|_{\Omega}^{2} .
\end{aligned}
$$

The second property is about the ordering of generations. For a given bisection sequence $\mathcal{B}$, we define $b_{i}<b_{j}$ if $i<j$, which means bisection $b_{i}$ is performed before $b_{j}$. The generation sequence $\left\{g_{1}, \cdots, g_{N}\right\}$, however, is not necessary monotone increasing; there could exist $b_{i}<b_{j}$ but $g_{i}>g_{j}$. This happens for bisections driven by a posteriori error estimators in practice. Adaptive algorithms usually refine elements around a singularity region first, thereby creating many elements with large generations, and later they refine coarse elements away from the singularity. This mixture of generations is the main difficulty for the analysis 
of multilevel methods on adaptive grids. We now prove the following quasi-monotonicity property of generations restricted to a fixed bisection patch.

Lemma 3.5 (Quasi-monotonicity). Let $\mathcal{T}_{N} \in \mathbb{T}\left(\mathcal{T}_{0}\right)$ be $\mathcal{T}_{N}=\mathcal{T}_{0}+\mathcal{B}$, where $\mathcal{B}=$ $\left\{b_{1}, \cdots, b_{N}\right\}$ is a compatible bisection sequence. For any $j>i$ and $\stackrel{\circ}{\tilde{\omega}}_{j} \cap \stackrel{\circ}{\tilde{\omega}}_{i} \neq \varnothing$, we have

$$
g_{j} \geq g_{i}-g_{0}
$$

where $g_{0}>0$ is an integer depending only on the shape regularity of $\mathcal{T}_{0}$.

Proof. Since $\stackrel{\circ}{\tilde{\omega}}_{j} \cap \stackrel{\circ}{\tilde{\omega}}_{i} \neq \varnothing$, there must be elements $\tau_{j} \in \mathcal{R}_{p_{j}} \cup \mathcal{R}_{p_{l_{j}}} \cup \mathcal{R}_{p_{r_{j}}}$ and $\tau_{i} \in \mathcal{R}_{p_{i}} \cup \mathcal{R}_{p_{l_{i}}} \cup \mathcal{R}_{p_{r_{i}}}$ such that $\stackrel{\circ}{\tau_{j}} \cap \stackrel{\circ}{\tau_{i}} \neq \varnothing$. Since we consider triangulations in $\mathbb{T}\left(\mathcal{T}_{0}\right)$, the intersection $\tau_{j} \cap \tau_{i}$ is still a simplex. When $b_{j}$ is performed, only $\tau_{j}$ exists in the current mesh. Thus $\tau_{j}=\tau_{j} \cap \tau_{i} \subseteq \tau_{i}$ and $g_{\tau_{j}} \geq g_{\tau_{i}}$.

Shape regularity implies the existence of a constant $g_{0}$ only depending on $\mathcal{T}_{0}$ such that

$$
g_{j}+g_{0} / 2 \geq g_{\tau_{j}} \geq g_{\tau_{i}} \geq g_{i}-g_{0} / 2
$$

and (3.12) follows.

A key practical issue is to find a decomposition of a bisection grid. We refer to Chen and Zhang [22] and Chen [19] for a vertex-oriented coarsening algorithm and the application to multilevel preconditioners and multigrid methods.

4. Space Decomposition on Bisection Grids. We give a space decomposition for Lagrange finite element spaces on bisection grids. Given a conforming mesh $\mathcal{T}$ of the domain $\Omega \subset \mathbb{R}^{d}$ and an integer $m \geq 1$, the $m$-th order finite element space on $\mathcal{T}$ is defined as follows:

$$
\mathcal{V}\left(\mathcal{P}_{m}, \mathcal{T}\right):=\left\{v \in H_{0}^{1}(\Omega):\left.v\right|_{\tau} \in \mathcal{P}_{m}(\tau) \text { for all } \tau \in \mathcal{T}\right\} .
$$

We restrict ourselves to bisection grids in $\mathbb{T}\left(\mathcal{T}_{0}\right)$ satisfying (B1) and (B2). Therefore by Theorem 3.1, for any $\mathcal{T}_{N} \in \mathbb{T}\left(\mathcal{T}_{0}\right)$, there exists a compatible bisection sequence $\mathcal{B}=$ $\left\{b_{1}, \cdots, b_{N}\right\}$ such that

$$
\mathcal{T}_{N}=\mathcal{T}_{0}+\mathcal{B}
$$

We give a decomposition of the finite element space $\mathcal{V}:=\mathcal{V}\left(\mathcal{P}_{m}, \mathcal{T}_{N}\right)$ using this decomposition of $\mathcal{T}_{N}$. If $\mathcal{T}_{i}=\mathcal{T}_{0}+\left\{b_{1}, \cdots, b_{i}\right\}$, let $\phi_{i, p} \in \mathcal{V}\left(\mathcal{P}_{1}, \mathcal{T}_{i}\right)$ denote the linear nodal basis at a vertex $p \in \mathcal{N}\left(\mathcal{T}_{i}\right)$. Let $\stackrel{\circ}{\mathcal{N}}\left(\mathcal{T}_{i}\right)$ denote the set of interior vertices of the triangulation $\mathcal{T}_{i}$. We define the sub-spaces

$$
\mathcal{V}_{0}:=\mathcal{V}\left(\mathcal{P}_{1}, \mathcal{T}_{0}\right), \text { and } \mathcal{V}_{i}:=\operatorname{span}\left\{\phi_{i, q}: q \in\left\{p_{i}, p_{l_{i}}, p_{r_{i}}\right\} \cap \stackrel{\circ}{\mathcal{N}}\left(\mathcal{T}_{i}\right)\right\}
$$

Since the basis functions of $\mathcal{V}_{i}, i=0, \ldots, N$, are piecewise linear polynomials on $\mathcal{T}_{N}$, we know $\mathcal{V}_{i} \subseteq \mathcal{V}$. Let $\left\{\phi_{p}: p \in \Lambda\right\}$ be a basis of $\mathcal{V}\left(\mathcal{P}_{m}, \mathcal{T}_{N}\right)$ such that $v=\sum_{p \in \Lambda} v(p) \phi_{p}$ for all $v \in \mathcal{V}\left(\mathcal{P}_{m}, \mathcal{T}_{N}\right)$, where $\Lambda$ is the set of indices. For example, for quadratic element spaces, $\Lambda$ consists of interior vertices and middle points of interior edges. We define $\mathcal{V}_{p}=\operatorname{span}\left\{\phi_{p}\right\}$ and end up with the following space decomposition:

$$
\mathcal{V}=\sum_{p \in \Lambda} \mathcal{V}_{p}+\sum_{i=0}^{N} \mathcal{V}_{i}
$$

The space decomposition (4.2) can be thought of as a decomposition into frequencies. The local mesh sizes $h_{i}$ and $h_{p}$ are the "wave lengths". In each subspace $\mathcal{V}_{i}$, the energy norm is equivalent to a scaling of the $L^{2}$-norm:

$$
h_{i}^{-2}\left\|v_{i}\right\|^{2} \lesssim\left\|v_{i}\right\|_{A}=\left|v_{i}\right|_{1} \lesssim h_{i}^{-2}\left\|v_{i}\right\|^{2}, \quad \text { for all } v_{i} \in \mathcal{V}_{i}, i=0, \cdots, J
$$


the same holds for functions in $\mathcal{V}_{p}$. The first inequality is just a scaled Poincaré inequality since $\mathcal{V}_{i} \subset H_{0}^{1}\left(\tilde{\omega}_{i}\right)$. The second inequality in (4.3) is the well known inverse inequality for finite element functions.

We next verify that the space decomposition (4.2) satisfies the assumptions (A1) and (A2).

4.1. Stable Decomposition: Proof of (1.6). The purpose of this section is to discuss and give a full proof of the following decomposition.

THEOREM 4.1 (Space decomposition over graded meshes). For any $v \in \mathcal{V}$, there exist $v_{p}, p \in \Lambda$, and $v_{i} \in \mathcal{V}_{i}, i=0, \cdots, N$ such that $v=\sum_{p \in \Lambda} v_{p}+\sum_{i=0}^{N} v_{i}$ and

$$
\sum_{p \in \Lambda}\left\|v_{p}\right\|_{A}^{2}+\sum_{i=0}^{N}\left\|v_{i}\right\|_{A}^{2} \lesssim\|v\|_{A}^{2} .
$$

An important ingredient of the proof is to use the Scott-Zhang quasi-interpolation operator [50]

$$
\mathcal{I}_{\mathcal{T}}: H_{0}^{1}(\Omega) \mapsto \mathcal{V}\left(\mathcal{P}_{1}, \mathcal{T}\right)
$$

for a conforming triangulation $\mathcal{T}$; see also Oswald [45]. For any interior vertex $p \in \mathcal{N}(\mathcal{T})$, we choose a $\tau_{p} \subset \mathcal{R}_{p}$. Let $\left\{\lambda_{\tau_{p}, i}: i=1, \cdots, d+1\right\}$ be the barycentric coordinates of $\tau_{p}$ which span $\mathcal{P}_{1}\left(\tau_{p}\right)$. We construct the $L^{2}$-dual basis $\Theta\left(\tau_{p}\right)=\left\{\theta_{\tau_{p}, i}: i=1, \cdots, d+1\right\}$ of $\left\{\lambda_{\tau_{p}, i}: i=1, \cdots, d+1\right\}$. If $\theta_{p} \in \Theta\left(\tau_{p}\right)$ is the dual basis function so that $\int_{\tau_{p}} \theta_{p} v \mathrm{~d} x=v(p)$, for all $v \in \mathcal{P}_{1}\left(\tau_{p}\right)$, we then define

$$
\mathcal{I}_{\mathcal{T}} v=\sum_{p \in \mathcal{N}(\mathcal{T})}\left(\int_{\tau_{p}} \theta_{p} v \mathrm{~d} x\right) \phi_{p},
$$

and note that $\mathcal{I}_{\mathcal{T}} v(p)=\int_{\tau_{p}} \theta_{p} v \lesssim h_{p}^{-2}\|v\|_{\tau_{p}}$. The operator $\mathcal{I}_{\mathcal{T}}$ preserves homogeneous Dirichlet conditions as well as the space of piecewise linear functions, and satisfies the following estimate and stability [50,45]

$$
\begin{gathered}
\left|\mathcal{I}_{\mathcal{T}} v\right|_{1}+\left\|h^{-1}\left(v-\mathcal{I}_{\mathcal{T}} v\right)\right\| \lesssim|v|_{1}, \\
h_{i}^{d}\left|\mathcal{I}_{\mathcal{T}} v\left(p_{i}\right)\right|^{2} \lesssim\|v\|_{\tau_{p_{i}}}^{2},
\end{gathered}
$$

with $h_{i}$ the size of $\tau_{p_{i}}$.

Given $v \in \mathcal{V}\left(\mathcal{P}_{m}, \mathcal{T}_{N}\right)$, we let $u=\mathcal{I}_{N} v$ and decompose $v=u+(v-u)$, where $\mathcal{I}_{N}: \mathcal{V}\left(\mathcal{P}_{m}, \mathcal{T}_{N}\right) \rightarrow \mathcal{V}\left(\mathcal{P}_{1}, \mathcal{T}_{N}\right)$ is the Scott-Zhang operator. We next give a multilevel decomposition of $u$ using a sequence of quasi-interpolations of Scott-Zhang type

$$
\mathcal{J}_{i}: \mathcal{V}\left(\mathcal{P}_{1}, \mathcal{T}_{N}\right) \rightarrow \mathcal{V}\left(\mathcal{P}_{1}, \mathcal{T}_{i}\right), \quad \text { for } i=0: N .
$$

First, we let $\mathcal{J}_{0}: \mathcal{V}\left(\mathcal{P}_{1}, \mathcal{T}_{N}\right) \rightarrow \mathcal{V}_{0}$ be a quasi-interpolation operator with values in the coarsest space $\mathcal{V}_{0}$. If we already have $\mathcal{J}_{i-1}: \mathcal{V}\left(\mathcal{P}_{1}, \mathcal{T}_{N}\right) \rightarrow \mathcal{V}\left(\mathcal{P}_{1}, \mathcal{T}_{i-1}\right)$, we recall that $\mathcal{T}_{i}=\mathcal{T}_{i-1}+b_{i}$ and exploit the fact that the bisection $b_{i}$ only changes the local patches of the two end points of the refinement edge $e_{i} \in \mathcal{E}\left(\mathcal{T}_{i-1}\right)$. We then define $\mathcal{J}_{i} u\left(p_{i}\right)$ at the newly added vertex $p_{i} \in \mathcal{N}^{\mathcal{N}}\left(\mathcal{T}_{i}\right)$ using a simplex $\tau_{i}$ newly created by the bisection $b_{i}$, namely $\tau_{i} \subset \omega_{i}$. If $p$ is any other vertex and $\tau_{p} \in \mathcal{T}_{i-1}$ is the simplex used to define $\mathcal{J}_{i-1} u(p)$, then we define $\mathcal{J}_{i} u(p)$ according to the following rules: 
1. if $\tau_{p} \subset \omega_{p}\left(\mathcal{T}_{i}\right)$ we keep the nodal value, i.e., $\mathcal{J}_{i} u(p)=\mathcal{J}_{i-1} u(p)$;

2. otherwise we choose a new $\tau_{p} \subset \omega_{p}\left(\mathcal{T}_{i}\right) \cap \omega_{p}\left(\mathcal{T}_{i-1}\right)$ to define $\mathcal{J}_{i} u(p)=\int_{\tau_{p}} \theta_{p} u$.

This construction guarantees that, in either case, the simplex $\tau_{p}$ satisfies (see Figure 4.1 for $d=2)$

$$
\tau_{p} \subset \omega_{p}\left(\mathcal{T}_{i}\right) \text { for all } p \in \stackrel{\circ}{\mathcal{N}}\left(\mathcal{T}_{i}\right) .
$$

Moreover, the slicing operator $\mathcal{J}_{i}-\mathcal{J}_{i-1}$ verifies the crucial property

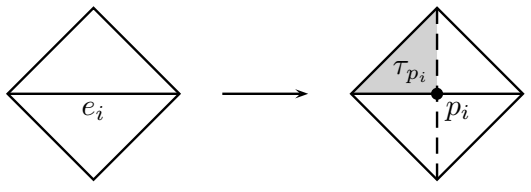

(a) Simplex to define $\left(\mathcal{J}_{i} u\right)\left(p_{i}\right)$

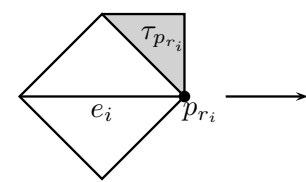

(c) Simplex to define $\left(\mathcal{J}_{i} u\right)\left(p_{r_{i}}\right)$

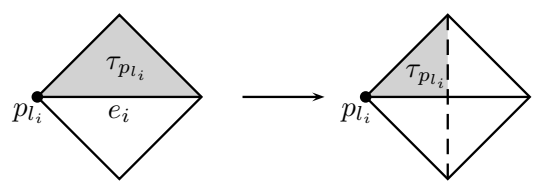

(b) Simplex to define $\left(\mathcal{J}_{i} u\right)\left(p_{l_{i}}\right)$

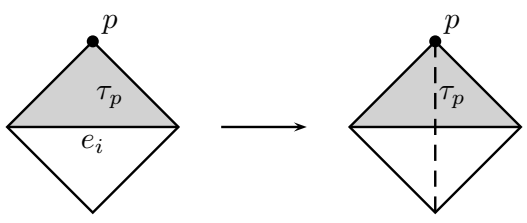

(d) Simplex to define $\left(\mathcal{J}_{i} u\right)(p)$

FIG. 4.1. Update of nodal values $\mathcal{J}_{i-1} u$ to yield $\mathcal{J}_{i} u$ : the element $\tau_{p}$ chosen to perform the averaging that gives $\left(\mathcal{J}_{i} u\right)(p)$ must belong to $\omega_{p}\left(\mathcal{T}_{i}\right)$. This implies $\left(\mathcal{J}_{i}-\mathcal{J}_{i-1}\right) u(p) \neq 0$ possibly for $p=p_{i}, p_{l_{i}}, p_{r_{i}}$ and $=0$ otherwise, and $\tau_{p}$ belongs to $\omega_{i}$ (the patch of $p_{i}$ in $\mathcal{T}_{i}$ ).

$$
v_{i}:=\left(\mathcal{J}_{i}-\mathcal{J}_{i-1}\right) u \in \mathcal{V}_{i} \quad \text { for all } 1 \leq i \leq N,
$$

because $\left(\mathcal{J}_{i}-\mathcal{J}_{i-1}\right) u(p)=0$ for $p \in \mathcal{N}\left(\mathcal{T}_{i}\right), p \neq p_{i}, p_{l_{i}}$ or $p_{r_{i}}$. Furthermore a closer look reveals that

if $\left(\mathcal{J}_{i}-\mathcal{J}_{i-1}\right) u(p) \neq 0$, then the elements $\tau_{p}$ used to define both $\mathcal{J}_{i}(p)$ or

$\mathcal{J}_{i-1}(p)$ are inside the patch $\omega_{i}$ of $p_{i}$ in $\mathcal{T}_{i}$. Figure 4.1 depicts four possible

configurations for $d=2$.

We stress that, in general $\mathcal{J}_{N} u \neq u=\mathcal{I}_{N} v$ because the simplex used to define nodal values of $\mathcal{J}_{N} u$ may not be in the finest mesh $\mathcal{T}_{N}$ but in $\mathcal{T}_{N-1}$ instead (see Figure 4.1(d)). Nevertheless, the difference $v-\mathcal{J}_{N} u=\left(u-\mathcal{J}_{N} u\right)+(v-u)$ is of high frequency in the finest mesh $\mathcal{T}_{N}$. We will exploit this fact.

Let $v-\mathcal{J}_{N} u=\sum_{p \in \Lambda} v_{p}$ be the nodal basis decomposition in $\mathcal{T}_{N}$. We then write

$$
v=\sum_{p \in \Lambda} v_{p}+\sum_{i=0}^{N} v_{i}, \quad v_{i} \in \mathcal{V}_{i},
$$

where $v_{i}=\left(\mathcal{J}_{i}-\mathcal{J}_{i-1}\right) u$ and for convenience we define $\mathcal{J}_{-1} u:=0$; thus $\mathcal{J}_{N} u=\sum_{i=0}^{N} v_{i}$.

To prove that the decomposition (4.10) is stable we first study the high frequency component $\sum_{p \in \Lambda} v_{p}$. According (4.5) and (4.7), we get

$$
\sum_{p \in \Lambda} h_{p}^{-2}\left\|v_{p}\right\|^{2} \lesssim\left\|h^{-1}\left(v-\mathcal{J}_{N} u\right)\right\|^{2} \lesssim\left\|h^{-1}\left(v-\mathcal{I}_{N} v\right)\right\|^{2}+\left\|h^{-1}\left(u-\mathcal{J}_{N} u\right)\right\|^{2} \lesssim|v|_{1}^{2}
$$


We next prove that the decomposition $\mathcal{J}_{N} u=\sum_{i=0}^{N}\left(\mathcal{J}_{i}-\mathcal{J}_{i-1}\right) u$ is stable. For this purpose, we consider an auxiliary decomposition over uniformly refined meshes $\overline{\mathcal{T}}_{k}$ of $\mathcal{T}_{0}$, and denote by $\overline{\mathcal{V}}_{k}=\mathcal{V}\left(\mathcal{P}_{1}, \overline{\mathcal{T}}_{k}\right)$ for $0 \leq k \leq L$. We choose $L=\max _{\tau \in \mathcal{T}_{N}} g_{\tau}$ so that $\mathcal{V}\left(\mathcal{P}_{1}, \mathcal{T}_{N}\right) \subseteq \overline{\mathcal{V}}_{L}$ and state the following well-known stable decomposition for the space $\overline{\mathcal{V}}_{L}=\sum_{k=0}^{L} \overline{\mathcal{V}}_{k}[61,43,44,13]$.

LEMMA 4.2 (Stable decomposition for quasi-uniform meshes). For any $\bar{v} \in \overline{\mathcal{V}}_{L}$, let $\bar{v}_{k}=\left(\bar{Q}_{k}-\bar{Q}_{k-1}\right) \bar{v}$ for $k=0, \cdots$, L. Then $\bar{v}=\sum_{k=0}^{L} \bar{v}_{k}$ is a stable decomposition in the sense that

$$
\sum_{k=0}^{L} \bar{h}_{k}^{-2}\left\|\bar{v}_{k}\right\|^{2} \lesssim|\bar{v}|_{1}^{2}
$$

We apply the slicing operator $\mathcal{J}_{i}-\mathcal{J}_{i-1}$ to this decomposition with $\bar{v}=\mathcal{J}_{N} u \in \overline{\mathcal{V}}_{L}$. If $g_{i}$ is the generation of bisection $b_{i}$ and $k \leq g_{i}-1$, then $\bar{v}_{k}$ is piecewise linear in $\omega_{e_{i}}$ (the patch of refinement edge $e_{i}$ ) and $\left(\mathcal{J}_{i}-\mathcal{J}_{i-1}\right) \bar{v}_{k}=0$ according to (4.9), because $\mathcal{J}_{i}$ preserves piecewise linear functions. So the slicing operator detects frequencies higher than or equal to $g_{i}$, namely,

$$
v_{i}=\left(\mathcal{J}_{i}-\mathcal{J}_{i-1}\right) \sum_{l=g_{i}}^{L} \bar{v}_{l} .
$$

Employing properties (4.8), (4.7) and (4.6), in this order, we infer that

$$
\left\|v_{i}\right\|_{\widetilde{\omega}_{i}}^{2} \lesssim h_{i}^{d}\left[v_{i}\left(p_{i}\right)^{2}+v_{i}\left(p_{l_{i}}\right)^{2}+v_{i}\left(p_{r_{i}}\right)^{2}\right] \lesssim\left\|\sum_{l=g_{i}}^{L} \bar{v}_{l}\right\|_{\omega_{i}}^{2},
$$

because the simplices used to define nonzero values of $v_{i}\left(p_{i}\right), v_{i}\left(p_{l_{i}}\right)$ or $v_{i}\left(p_{r_{i}}\right)$ are inside $\omega_{i}$ according to (4.9). This explains why the domain of integration on the right-hand side is $\omega_{i}$ instead of $\tilde{\omega}_{i}$.

Since distinct bisections patches with the same generation are weakly disjoint (Lemma 3.4), namely $\stackrel{\circ}{\omega}_{i} \cap \stackrel{\circ}{\omega_{j}}=\varnothing$ if $g_{i}=g_{j}$, we deduce

$$
\sum_{g_{i}=k}\left\|v_{i}\right\|^{2}=\sum_{g_{i}=k}\left\|v_{i}\right\|_{\tilde{\omega}_{i}}^{2} \lesssim \sum_{g_{i}=k}\left\|\sum_{l=g_{i}}^{L} \bar{v}_{l}\right\|_{\omega_{i}}^{2} \leq\left\|\sum_{l=k}^{L} \bar{v}_{l}\right\|_{\Omega}^{2}=\sum_{l=k}^{L}\left\|\bar{v}_{l}\right\|^{2},
$$

where in the last step we have used that $\left\{\bar{v}_{l}\right\}$ are $L^{2}$-orthogonal.

To proceed further we need the following elementary result, which can be found in [25].

LEMMA 4.3 (Discrete Hardy inequality). If the non-negative sequences $\left\{a_{k}\right\}_{k=0}^{L},\left\{b_{k}\right\}_{k=0}^{L}$ satisfy

$$
b_{k} \leq \sum_{l=k}^{L} a_{l}, \quad \text { for all } k \geq 0,
$$

then for any $s \in(0,1)$ we have

$$
\sum_{k=0}^{L} s^{-k} b_{k} \leq \frac{1}{1-s} \sum_{k=0}^{L} s^{-k} a_{k}
$$


Proof. Since

$$
\sum_{k=0}^{L} s^{-k} b_{k} \leq \sum_{k=0}^{L} \sum_{l=k}^{L} s^{-k} a_{l}=\sum_{l=0}^{L} \sum_{k=0}^{l} s^{-k} a_{l}=\sum_{l=0}^{L} s^{-l} a_{l} \sum_{k=0}^{l} s^{l-k},
$$

and $s<1$, the geometric series is bounded by $1 /(1-s)$. This concludes the proof. $\square$

Applying Lemma 4.3 with $s=\gamma=(1 / 2)^{1 / d}$ to $a_{k}=\left\|\bar{v}_{k}\right\|^{2}$ and $b_{k}=\sum_{g_{i}=k}\left\|v_{i}\right\|^{2}$, and recalling that $h_{k} \approx \gamma^{g_{k}}$ according to (3.8), we obtain

$$
\sum_{k=0}^{L} \bar{h}_{k}^{-2} \sum_{g_{i}=k}\left\|v_{i}\right\|^{2} \lesssim \sum_{k=0}^{L} \bar{h}_{k}^{-2}\left\|\bar{v}_{k}\right\|^{2},
$$

and thus from the stable decomposition of Lemma 4.2 for uniform refinement, we conclude

$$
\sum_{i=0}^{N} h_{i}^{-2}\left\|v_{i}\right\|^{2}=\sum_{k=0}^{L} \bar{h}_{k}^{-2} \sum_{g_{i}=k}\left\|v_{i}\right\|^{2} \lesssim \sum_{k=0}^{L} \bar{h}_{k}^{-2}\left\|\bar{v}_{k}\right\|^{2} \lesssim\left|\mathcal{J}_{N} u\right|_{1}^{2} \lesssim|v|_{1}^{2}
$$

Finally, combining (4.11), (4.14) and invoking (4.3), we get the asserted estimate (4.4).

REMARK 4.4 (Spaces decompositions for traditional smoothing). Consider piecewise linear finite element spaces, i.e. $m=1$. If we define $\tilde{\mathcal{V}}_{i}=\operatorname{span}\left\{\phi_{i, q}, q \in \omega_{p_{i}} \cap \stackrel{\circ}{\mathcal{N}}\left(\mathcal{T}_{i}\right)\right\}$ to be the space spanned by the hat basis functions associated with node $p_{i}$ and all neighboring nodes, then in the construction of the quasi-interpolation operators $\mathcal{J}_{i}$ we can always choose a simplex $\tau_{p}$ in the star of $\omega_{p}$ for each vertex $p$, whence the difference $\left(\mathcal{J}_{i}-\mathcal{J}_{i-1}\right) u \in \tilde{\mathcal{V}}_{i}$ and $\mathcal{J}_{N} u=u$. Consequently we can prove that the space decomposition $\mathcal{V}=\sum_{i=0}^{N} \tilde{\mathcal{V}}_{i}$ is stable. The corresponding subspace correction methods require smoothing in the new node and all neighboring nodes, but an additional fine grid smoothing is not necessary.

Similarly for quadratic and higher elements, i.e. $m \geq 2$, one can include all degree of freedom (dof) inside the bisection patch $\omega_{i}$ into the subspace $\tilde{\mathcal{V}}_{i}$ and obtain a stable decomposition. The corresponding local multigrid methods then requires smoothing on all dof inside $\omega_{i}$; see [39].

4.2. Strengthened Cauchy-Schwarz Inequality: Proof of (1.7). In this section we establish the strengthened Cauchy-Schwarz (SCS) inequality (1.7) for the space decomposition $\sum_{i=0}^{N} \mathcal{V}_{i}$. We first present a SCS inequality for uniform refinement.

LEMma 4.5 (SCS inequality for quasi-uniform meshes; see Lemma 4.26 in [63]). For any $u_{i} \in \overline{\mathcal{V}}_{i}, v_{j} \in \overline{\mathcal{V}}_{j}, j \geq i$, we have

$$
\left(u_{i}, v_{j}\right)_{A} \lesssim \gamma^{j-i}\left|u_{i}\right|_{1} h_{j}^{-1}\left\|v_{j}\right\|_{0},
$$

where $\gamma<1$ is a constant such that $h_{i} \bar{\sim} \gamma^{2 i}$.

Proof. Let us first prove the inequality on one element $\tau \in \mathcal{T}_{i}$. Using integration by parts, Cauchy-Schwarz inequality, and inverse inequality, we have

$$
\begin{aligned}
\int_{\tau} \nabla u_{i} \cdot \nabla v_{j} \mathrm{~d} x & =\int_{\partial \tau} \frac{\partial u_{i}}{\partial n} v_{j} d s \lesssim\left\|\nabla u_{i}\right\|_{0, \partial \tau}\left\|v_{j}\right\|_{0, \partial \tau} \lesssim h_{i}^{-1 / 2}\left\|\nabla u_{i}\right\|_{0, \tau} h_{j}^{-1 / 2}\left\|v_{j}\right\|_{0, \tau} \\
& \lesssim\left(\frac{h_{j}}{h_{i}}\right)^{1 / 2}\left|u_{i}\right|_{1, \tau} h_{j}^{-1}\left\|v_{j}\right\|_{0, \tau} \approx \gamma^{j-i}\left|u_{i}\right|_{1, \tau} h_{j}^{-1}\left\|v_{j}\right\|_{0, \tau} .
\end{aligned}
$$


Adding over $\tau \in \mathcal{T}_{i}$, and using Cauchy-Schwarz inequality again, yields

$$
\begin{aligned}
\left(\nabla u_{i}, \nabla v_{j}\right) & =\sum_{\tau \in \mathcal{T}_{i}}\left(\nabla u_{i}, \nabla v_{j}\right)_{\tau} \lesssim \gamma^{j-i} h_{j}^{-1} \sum_{\tau \in \mathcal{T}_{i}}\left|u_{i}\right|_{1, \tau}\left\|v_{j}\right\|_{0, \tau} \\
& \lesssim \gamma^{j-i} h_{j}^{-1}\left(\sum_{\tau \in \mathcal{T}_{i}}\left|u_{i}\right|_{1, \tau}^{2}\right)^{1 / 2}\left(\sum_{\tau \in \mathcal{T}_{i}}\left\|v_{j}\right\|_{0, \tau}^{2}\right)^{1 / 2} \\
& =\gamma^{j-i}\left|u_{i}\right|_{1} h_{j}^{-1}\left\|v_{j}\right\|_{0},
\end{aligned}
$$

which is the asserted estimate. $\square$

THEOREM 4.6 (SCS inequality for graded meshes). For any $u_{i}, v_{i} \in \mathcal{V}_{i}, i=0, \cdots, N$, we have

$$
\left|\sum_{i=0}^{N} \sum_{j=i+1}^{N}\left(u_{i}, v_{j}\right)_{A}\right| \lesssim\left(\sum_{i=0}^{N}\left\|u_{i}\right\|_{A}^{2}\right)^{1 / 2}\left(\sum_{i=0}^{N}\left\|v_{i}\right\|_{A}^{2}\right)^{1 / 2}
$$

Proof. We resort to the concept of generation to rewrite several sums in terms of uniform refinements. The proof is divided into four steps.

1 For a fixed index $i \in[1, N]$, we denote by

$$
n(i)=\left\{j>i: \stackrel{\circ}{\tilde{\omega}}_{j} \cap \stackrel{\check{\omega}}{i}_{i} \neq \varnothing\right\} \text { and } w_{k}^{i}=\sum_{j \in n(i), g_{j}=k} v_{j} .
$$

We observe that $w_{k}^{i} \in \overline{\mathcal{V}}_{k}$ and $k=g_{j} \geq g_{i}-g_{0}$ whereas $u_{i} \in \overline{\mathcal{V}}_{g_{i}+g_{0}}$ according to Lemma 3.5. For any $\tau \subset \widetilde{\omega}_{i}$, we apply Lemma 4.5 over $\tau$ to $u_{i}$ and $w_{k}^{i}$ to obtain

$$
\left(u_{i}, w_{k}^{i}\right)_{A, \tau} \lesssim \gamma^{k-g_{i}}\left\|u_{i}\right\|_{A, \tau} \bar{h}_{k}^{-1}\left\|w_{k}^{i}\right\|_{\tau} .
$$

Then

$$
\begin{aligned}
\left(u_{i}, w_{k}^{i}\right)_{A, \widetilde{\omega}_{i}}=\sum_{\tau \subset \widetilde{\omega}_{i}}\left(u_{i}, w_{k}^{i}\right)_{A, \tau} & \lesssim \gamma^{k-g_{i}} \sum_{\tau \subset \widetilde{\omega}_{i}}\left\|u_{i}\right\|_{A, \tau} \bar{h}_{k}^{-1}\left\|w_{k}^{i}\right\|_{\tau} \\
& \lesssim \gamma^{k-g_{i}}\left\|u_{i}\right\|_{A, \widetilde{\omega}_{i}} \bar{h}_{k}^{-1}\left(\sum_{\tau \subset \widetilde{\omega}_{i}}\left\|w_{k}^{i}\right\|_{\tau}^{2}\right)^{1 / 2} .
\end{aligned}
$$

Since $v_{j}$ 's with the same generation $g_{j}=k$ have supports supp $v_{j} \subset \widetilde{\omega}_{j}$ with finite overlap (see Lemma 3.4), we infer that $\left\|w_{k}^{i}\right\|_{\tau}^{2} \lesssim \sum_{j \in n(i), g_{j}=k}\left\|v_{j}\right\|_{\tau}^{2} \leq \sum_{g_{j}=k}\left\|v_{j}\right\|_{\tau}^{2}$ and

$$
\left(u_{i}, w_{k}^{i}\right)_{A, \widetilde{\omega}_{i}} \lesssim \gamma^{k-g_{i}}\left\|u_{i}\right\|_{A, \widetilde{\omega}_{i}} \bar{h}_{k}^{-1}\left(\sum_{g_{j}=k}\left\|v_{j}\right\|_{0, \widetilde{\omega}_{i}}^{2}\right)^{1 / 2} .
$$

2 We fix $u_{i}$ and consider

$$
\begin{aligned}
\left|\left(u_{i}, \sum_{j=i+1}^{N} v_{j}\right)_{A}\right| & =\left|\left(u_{i}, \sum_{j \in n(i)} v_{j}\right)_{A, \widetilde{\omega}_{i}}\right| \\
& =\left|\left(u_{i}, \sum_{k=g_{i}-g_{0}}^{L} \sum_{j \in n(i), g_{j}=k} v_{j}\right)_{A, \widetilde{\omega}_{i}}\right| \leq \sum_{k=g_{i}-g_{0}}^{L}\left|\left(u_{i}, w_{k}^{i}\right)_{A, \widetilde{\omega}_{i}}\right|,
\end{aligned}
$$


because $\left(u_{i}, v_{j}\right)=0$ for $k=g_{j}<g_{i}-g_{0}$ (Lemma 3.5). Since $k \geq 0$ and $k \geq g_{i}-g_{0}$, this is equivalent to $k \geq\left(g_{i}-g_{0}\right)^{+}=\max \left\{g_{i}-g_{0}, 0\right\}$, whence

$$
\left|\left(u_{i}, \sum_{j=i+1}^{N} v_{j}\right)_{A}\right| \lesssim \sum_{k=\left(g_{i}-g_{0}\right)^{+}}^{L} \gamma^{k-g_{i}}\left\|u_{i}\right\|_{A, \widetilde{\omega}_{i}} \bar{h}_{k}^{-1}\left(\sum_{g_{j}=k}\left\|v_{j}\right\|_{0, \widetilde{\omega}_{i}}^{2}\right)^{1 / 2} .
$$

3 We now sum over $i$ but keeping the generation $g_{i}=l \geq 0$ fixed:

$$
\begin{aligned}
\sum_{g_{i}=l}\left|\left(u_{i}, \sum_{j=i+1}^{N} v_{j}\right)_{A}\right| & \lesssim \sum_{k=\left(l-g_{0}\right)^{+}}^{L} \gamma^{k-l}\left\{\sum_{g_{i}=l}\left[\left\|u_{i}\right\|_{A, \widetilde{\omega}_{i}}\left(\bar{h}_{k}^{-2} \sum_{g_{j}=k}\left\|v_{j}\right\|_{\widetilde{\omega}_{i}}^{2}\right)^{1 / 2}\right]\right\} \\
& \lesssim \sum_{k=\left(l-g_{0}\right)^{+}}^{L} \gamma^{k-l}\left(\sum_{g_{i}=l}\left\|u_{i}\right\|_{A, \widetilde{\omega}_{i}}^{2}\right)^{1 / 2}\left(\bar{h}_{k}^{-2} \sum_{g_{i}=l} \sum_{g_{j}=k}\left\|v_{j}\right\|_{\widetilde{\omega}_{i}}^{2}\right)^{1 / 2} .
\end{aligned}
$$

In view of (3.11), due to the finite overlap property of patches $\widetilde{\omega}_{i}$ for generation $g_{i}=l$, we deduce

$$
\sum_{g_{i}=l}\left|\left(u_{i}, \sum_{j=i+1}^{N} v_{j}\right)_{A}\right| \lesssim \sum_{k=\left(l-g_{0}\right)^{+}}^{L} \gamma^{k-l}\left(\sum_{g_{i}=l}\left\|u_{i}\right\|_{A, \widetilde{\omega}_{i}}^{2}\right)^{1 / 2}\left(\bar{h}_{k}^{-2} \sum_{g_{j}=k}\left\|v_{j}\right\|^{2}\right)^{1 / 2} .
$$

4. We finally sum over all generations $0 \leq l \leq L$ to get

$$
\begin{aligned}
\sum_{l=0}^{L} \sum_{g_{i}=l}\left|\left(u_{i}, \sum_{j=i+1}^{N} v_{j}\right)_{A}\right| & \lesssim \sum_{l=0}^{L} \sum_{k=\left(l-g_{0}\right)^{+}}^{L} \gamma^{k-l}\left(\sum_{g_{i}=l}\left\|u_{i}\right\|_{A, \widetilde{\omega}_{i}}^{2}\right)^{1 / 2}\left(\bar{h}_{k}^{-2} \sum_{g_{j}=k}\left\|v_{j}\right\|^{2}\right)^{1 / 2} \\
& \lesssim\left(\sum_{l=0}^{L} \sum_{g_{i}=l}\left\|u_{i}\right\|_{A, \widetilde{\omega}_{i}}^{2}\right)^{1 / 2}\left(\sum_{k=0}^{L} \bar{h}_{k}^{-2} \sum_{g_{j}=k}\left\|v_{j}\right\|^{2}\right)^{1 / 2}
\end{aligned}
$$

where we have used the estimate

$$
\sum_{i, j=1}^{n} \gamma^{|i-j|} x_{i} x_{j} \leq \frac{2}{1-\gamma}\left(\sum_{i=1}^{n} x_{i}^{2}\right)^{1 / 2}\left(\sum_{i=1}^{n} y_{i}^{2}\right)^{1 / 2} \forall\left(x_{i}\right)_{i=1}^{n},\left(y_{i}\right)_{i=1}^{n} \in \mathbb{R}^{n} .
$$

Since $\sum_{i=0}^{N}=\sum_{l=0}^{L} \sum_{g_{i}=l}$ and $\bar{h}_{k}=h_{j}$ for $k=g_{j}$, using the local norm equivalence (4.3), we end up with the desired estimate (4.15).

REMARK 4.7 (Traditional smoothers). A SCS inequality similar to (4.15) can be proved for the slightly larger spaces $\tilde{\mathcal{V}}_{i}$ of Remark 4.4. Using the stable decomposition of $\mathcal{V}=$ $\sum_{i=0}^{N} \tilde{\mathcal{V}}_{i}$, we can prove that local multigrid methods with traditional smoothers also converge uniformly for graded bisection meshes.

To apply the abstract convergence theory for SSC to the space decomposition $\mathcal{V}=$ $\sum_{p \in \Lambda} \mathcal{V}_{p}+\sum_{i=0}^{N} \mathcal{V}_{i}$, we still need to prove the following inequalities.

THEOREM 4.8 (SCS between high frequency and multilevel decomposition). For any $v_{p} \in \mathbb{V}_{p}$ and $u_{i} \in \mathbb{V}_{i}$, we have

$$
\left|\sum_{p \in \Lambda} \sum_{i=0}^{N}\left(v_{p}, u_{i}\right)_{A}\right| \lesssim\left(\sum_{p \in \Lambda}\left\|v_{p}\right\|_{A}^{2}\right)^{1 / 2}\left(\sum_{i=0}^{N}\left\|u_{i}\right\|_{A}^{2}\right)^{1 / 2},
$$


and

$$
\left|\sum_{p \in \Lambda} \sum_{q \in \Lambda, q>p}\left(u_{p}, v_{q}\right)_{A}\right| \lesssim\left(\sum_{p \in \Lambda}\left\|u_{p}\right\|_{A}^{2}\right)^{1 / 2}\left(\sum_{q \in \Lambda}\left\|v_{q}\right\|_{A}^{2}\right)^{1 / 2},
$$

where we assume there is an ordering in the set $\Lambda$.

Proof. By the Cauchy-Schwarz inequality and the finite overlap property of the stars $\omega_{p}$, we deduce

$$
\begin{aligned}
\left|\sum_{p \in \Lambda} \sum_{i=0}^{N}\left(v_{p}, u_{i}\right)_{A}\right| \lesssim \sum_{p \in \Lambda}\left(\left\|v_{p}\right\|_{A}\left\|\sum_{i=0}^{N} u_{i}\right\|_{A, \omega_{p}}\right) \\
\qquad\left(\sum_{p \in \Lambda}\left\|v_{p}\right\|_{A}^{2}\right)^{1 / 2}\left(\sum_{p \in \Lambda}\left\|\sum_{i=0}^{N} u_{i}\right\|_{A, \omega_{p}}^{2}\right)^{1 / 2} \\
\lesssim\left(\sum_{p \in \Lambda}\left\|v_{p}\right\|_{A}^{2}\right)^{1 / 2}\left\|\sum_{i=0}^{N} u_{i}\right\|_{A} .
\end{aligned}
$$

Combining this with the estimate

$$
\left\|\sum_{i=0}^{N} u_{i}\right\|_{A}^{2}=\left(\sum_{i=0}^{N} u_{i}, \sum_{i=0}^{N} u_{i}\right)_{A} \leq \sum_{i=0}^{N}\left\|u_{i}\right\|_{A}^{2}+2\left|\sum_{i=0}^{N} \sum_{j=i+1}^{N}\left(u_{i}, u_{j}\right)_{A}\right| \lesssim \sum_{i=0}^{N}\left\|u_{i}\right\|_{A}^{2},
$$

we then get (4.16). The proof of (4.17) is simpler and follows from mesh shape regularity. In fact, given $p \in \Lambda$, the cardinality of the set $\left\{q \in \Lambda: q>p\right.$ and $\left.\omega_{p} \cap \omega_{p} \neq \emptyset\right\}$ is uniformly bounded.

5. BPX Preconditioner and Multigrid on Graded Bisection Grids. We apply PSC and SSC to the space decomposition

$$
\mathcal{V}=\sum_{p \in \Lambda} \mathcal{V}_{p}+\sum_{i=0}^{N} \mathcal{V}_{i}
$$

and thus obtain multilevel preconditioners and V-cycle multigrid methods on graded bisection grids.

We first observe that we can use standard smoothers, e.g. Richardson, symmetric GaussSeidal or Jacobi, for the nodal basis decomposition. Since $\operatorname{dim} \mathcal{V}_{i}=3$, we have a three-point local smoother for multilevel decomposition and the total computational cost for subspace correction methods based on (5.1) is $C N$, with a relatively small constant $C$. In addition, the three-point local smoother simplifies the implementation of multilevel methods especially in dimensions higher than 2. For example, we only need to maintain an ordered vertex array with two parent vertices rather than a tree structure to store the hierarchical mesh structure.

BPX Preconditioner. We use the stable decomposition and SCS inequality to obtain the optimality of the resulting preconditioner.

THEOREM 5.1 (Optimality of BPX on graded bisection grids). If the preconditioner $B$ of (2.3) is based on the space decomposition (5.1) and SPD smoothers satisfying (2.12), then we have

$$
\kappa(B A) \lesssim 1
$$


Proof. Combine Theorems 4.1 and 4.6 with Theorem 2.4. $\square$ As mentioned above, standard smoothers such as Richardson, Jacobi, or symmetric Gauss-Seidel iterations satisfy (2.12) and therefore we have uniform convergence of PCG with additive preconditioner.

V-Cycle Multigrid. This method results from applying SSC to the space decomposition (5.1). A standard V-cycle loop reads as follows:

1. pre-smoothing in the finest space $\mathcal{V}\left(\mathcal{P}_{m}, \mathcal{T}_{N}\right)$;

2. multilevel smoothing in piecewise linear finite element spaces $\mathcal{V}_{i}$ for $i=N$ to 1 ;

3. approximate solving in the coarsest piecewise linear finite element space $\mathcal{V}_{0}$;

4. multilevel smoothing in piecewise linear finite element spaces $\mathcal{V}_{i}$ for $i=1$ to $N$;

5. post-smoothing in the finest space $\mathcal{V}\left(\mathcal{P}_{m}, \mathcal{T}_{N}\right)$.

We remark that the smoothing in the finest space $\mathcal{V}\left(\mathcal{P}_{m}, \mathcal{T}_{N}\right)$ is crucial to take care of high frequency modes which may not be seen by the multilevel splitting $\left\{\mathcal{V}_{i}\right\}_{i=1}^{N}$ of piecewise linear finite element spaces; see (4.4).

THEOREM 5.2 (Uniform convergence of V-cycle multigrid on graded bisection grids). The above V-cycle multigrid, based on the space decomposition (5.1) with smoothers satisfying the contraction property $(\mathbf{T})$, is uniformly convergent.

Proof. Combine Theorems 4.1 and 4.6 with Theorem 2.6.

\section{REFERENCES}

[1] B. Aksoylu, S. Bond, and M. Holst. An odyssey into local refinement and multilevel preconditioning III: Implementation and numerical experiments. SIAM J. Sci. Comput., 25(2):478-498, 2003.

[2] B. Aksoylu and M. Holst. Optimality of multilevel preconditioners for local mesh refinement in three dimensions. SIAM J. Numer. Anal., 44(3):1005-1025, 2006.

[3] D. N. Arnold, A. Mukherjee, and L. Pouly. Locally adapted tetrahedral meshes using bisection. SIAM J. Sci. Comput., 22(2):431-448, 2000.

[4] D. Bai and A. Brandt. Local mesh refinement multilevel techniques. SIAM J. Sci. Statist. Comput., 8:109-134, 1987.

[5] R. Bank. PLTMG: A Software Package for Solving Elliptic Partial Differential Equations Users' Guide 6.0. Society for Industrial and Applied Mathematics, 1990.

[6] R. E. Bank, T. Dupont, and H. Yserentant. The hierarchical basis multigrid method. Numer. Math., 52:427458, 1988.

[7] R. E. Bank, A. H. Sherman, and A. Weiser. Refinement algorithms and data structures for regular local mesh refinement. In Scientific Computing, pages 3-17. IMACS/North-Holland Publishing Company, Amsterdam, 1983.

[8] E. Bänsch. Local mesh refinement in 2 and 3 dimensions. Impact Comput. Sci. Engrg., 3:181-191, 1991.

[9] T. C. Biedl, P. Bose, E. D. Demaine, and A. Lubiw. Efficient algorithms for Petersen's matching theorem. In Symposium on Discrete Algorithms, pages 130-139, 1999.

[10] P. Binev, W. Dahmen, and R. DeVore. Adaptive finite element methods with convergence rates. Numer. Math., 97(2):219-268, 2004.

[11] F. Bornemann, B. Erdmann, and R. Kornhuber. Adaptive multilevel methods in three space dimensions. Int. J. for Numer. Meth. in Eng., 36(18):3187-3203, 1993.

[12] F. A. Bornemann. An adaptive multilevel approach to parabolic equations. III. 2D error estimation and multilevel preconditioning. Impact Comput. Sci. Engrg., 4(1):1-45, 1992.

[13] F. A. Bornemann and H. Yserentant. A basic norm equivalence for the theory of multilevel methods. Numer. Math., 64:455-476, 1993.

[14] J. H. Bramble and J. E. Pasciak. New estimates for multigrid algorithms including the V-cycle. Math. Comp., 60:447-471, 1993.

[15] J. H. Bramble, J. E. Pasciak, and J. Xu. Parallel multilevel preconditioners. Math. Comp., 55(191):1-22, 1990.

[16] J. H. Bramble and X. Zhang. The analysis of multigrid methods. In Handbook of numerical analysis, Vol. VII, pages 173-415. North-Holland, Amsterdam, 2000.

[17] A. Brandt. Multi-level adaptive solutions to boundary-value problems. Math. Comp., 31:333-390, 1977.

[18] J. M. Cascón, C. Kreuzer, R. H. Nochetto, and K. G. Siebert. Quasi-optimal convergence rate for an adaptive finite element method. SIAM J. Numer. Anal., 46(5):2524-2550, 2008. 
[19] L. Chen. $i$ FEM: an integrate finite element methods package in MATLAB. Technical Report, University of California at Irvine, 2009.

[20] L. Chen. Deriving the X-Z Identity from Auxiliary Space Method. In The Proceedings for 19th Conferences for Domain Decomposition Methods, 2010.

[21] L. Chen, M. Holst, J. Xu, and Y. Zhu. Local multilevel preconditioners for elliptic equations with jump coefficients on bisection grids. Submitted, 2010.

[22] L. Chen and C.-S. Zhang. A coarsening algorithm on adaptive grids by newest vertex bisection and its applications. J. Comp. Math., 28(6):767-789, 2010.

[23] D. Cho, J. Xu, and L. Zikatanov. New estimates for the rate of convergence of the method of subspace corrections. Numer. Math. Theor. Meth. Appl., 1:44-56, 2008.

[24] W. Dahmen and A. Kunoth. Multilevel preconditioning. Numer. Math., 63:315-344, 1992

[25] R. A. Devore and G. G. Lorentz. Constructive Approximation. Spring-Verlag, New York, NY, 1993.

[26] W. Dörfler. A convergent adaptive algorithm for Poisson's equation. SIAM J. Numer. Anal., 33:1106-1124, 1996.

[27] M. Griebel. Adaptive sparse grid multilevel methods for elliptic PDEs based on finite differences. Computing, 61(2):151-179, 1998. also as Proceedings Large-Scale Scientific Computations of Engineering and Environmental Problems, 7. June - 11. June, 1997, Varna, Bulgaria, Notes on Numerical Fluid Mechanics 62, Vieweg-Verlag, Braunschweig, M. Griebel, O. Iliev, S. Margenov and P. Vassilevski (editors).

[28] M. Griebel and P. Oswald. On additive Schwarz preconditioners for sparse grid discretization. Numer. Math., 66:449-464, 1994.

[29] M. Griebel and P. Oswald. On the abstract theory of additive and multiplicative Schwarz methods. Numer. Math., 70:163-180, 1995.

[30] M. Griebel and G. Zumbusch. Parallel adaptive subspace correction schemes with applications to elasticity. Comput. Methods Appl. Mech. Engrg., 184(2-4):303-332, 2000.

[31] R. Hiptmair and W. Zheng. Local Multigrid in H(curl). J. Comput. Math., 27(5):573-603, 2009.

[32] I. Kossaczký. A recursive approach to local mesh refinement in two and three dimensions. J. Comput. Math., 55:275-288, 1994.

[33] J. Maubach. Local bisection refinement for $n$-simplicial grids generated by reflection. SIAM J. Sci. Comput., 16(1):210-227, 1995.

[34] S. McCormick. Fast adaptive composite grid (FAC) methods: theory for the variational case. In Defect correction methods (Oberwolfach, 1983), volume 5 of Comput. Suppl., pages 115-121. Springer, Vienna, 1984.

[35] S. F. McCormick and J. W. Thomas. The fast adaptive composite grid (FAC) method for elliptic equations. Math. Comp., 46:439-456, 1986.

[36] K. Mekchay and R. H. Nochetto. Convergence of adaptive finite element methods for general second order linear elliptic PDE. SIAM J. Numer. Anal., 43(5):1803-1827, 2005.

[37] W. F. Mitchell. Unified Multilevel Adaptive Finite Element Methods for Elliptic Problems. PhD thesis, University of Illinois at Urbana-Champaign, 1988.

[38] W. F. Mitchell. A comparison of adaptive refinement techniques for elliptic problems. ACM Trans. Math. Softw. (TOMS) archive, 15(4):326 - 347, 1989.

[39] W. F. Mitchell. Optimal multilevel iterative methods for adaptive grids. SIAM J. Sci. Statist. Comput., 13:146167, 1992.

[40] P. Morin, R. H. Nochetto, and K. G. Siebert. Data oscillation and convergence of adaptive FEM. SIAM J. Numer. Anal., 38(2):466-488, 2000.

[41] P. Morin, R. H. Nochetto, and K. G. Siebert. Convergence of adaptive finite element methods. SIAM Rev., 44(4):631-658, 2002.

[42] R. H. Nochetto, K. G. Siebert, and A. Veeser. Theory of adaptive finite element methods: an introduction. In R. A. DeVore and A. Kunoth, editors, Multiscale, Nonlinear and Adaptive Approximation. Springer, 2009.

[43] P. Oswald. Norm equivalencies and multilevel Schwarz preconditioning for variational problems. Forschungsergebnisse Math-1-92, Fak. Math. Informatik, FSU Jena, 1992.

[44] P. Oswald. On discrete norm estimates related to multilevel preconditioners in the finite element method. In Constructive Theory of Functions, Proc. Int. Conf. Varna 1991, pages 203-214, Sofia, 1992. Bulg. Acad. Sci.

[45] P. Oswald. Multilevel Finite Element Approximation, Theory and Applications. Teubner Skripten zur Numerik. Teubner Verlag, Stuttgart, 1994.

[46] A. Plaza and G. F. Carey. Local refinement of simplicial grids based on the skeleton. Appl. Numer. Math., 32(2): 195-218, 2000.

[47] M. C. Rivara. Design and data structure for fully adaptive, multigrid finite element software. ACM Trans. Math. Soft., 10:242-264, 1984.

[48] M. C. Rivara. Mesh refinement processes based on the generalized bisection of simplices. SIAM J. Numer. Anal., 21:604-613, 1984. 
[49] A. Schmidt and K. G. Siebert. Design of Adaptive Finite Element Software: The Finite Element Toolbox ALBERTA, volume 42 of Lecture Notes in Computational Science and Engineering. Springer-Verlag, Berlin, 2005. The finite element toolbox ALBERTA, With 1 CD-ROM (Unix/Linux).

[50] R. Scott and S. Zhang. Finite element interpolation of nonsmooth functions satisfying boundary conditions. Math. Comp., 54:483-493, 1990.

[51] E. G. Sewell. Automatic Generation of Triangulations for Piecewise Polynomial Approximation. PhD thesis, Purdue Univ., West Lafayette, Ind., 1972.

[52] R. Stevenson. Stable three-point wavelet bases on general meshes. Numer. Math., V80(1):131-158, 1998.

[53] R. Stevenson. Optimality of a standard adaptive finite element method. Found. Comput. Math., 7(2):245-269, 2007.

[54] R. Stevenson. The completion of locally refined simplicial partitions created by bisection. Math. Comp., 77:227-241, 2008.

[55] C. T. Traxler. An algorithm for adaptive mesh refinement in $n$ dimensions. Computing, 59(2):115-137, 1997.

[56] P. S. Vassilevski and J. Wang. Stabilizing the hierarchical basis by approximate wavelets, I: Theory. Numer. Linear Alg. Appl., 4(2):103-126, 1997.

[57] P. S. Vassilevski and J. Wang. Stabilizing the hierarchical basis by approximate wavelets II: Implementation and numerical results. SIAM J. Sci. Comput., 20(2):490-514, 1998.

[58] R. Verfürth. A Review of A Posteriori Error Estimation and Adaptive Mesh Refinement Tecniques. B. G. Teubner, 1996.

[59] O. B. Widlund. Some Schwarz methods for symmetric and nonsymmetric elliptic problems. In D. E. Keyes, T. F. Chan, G. A. Meurant, J. S. Scroggs, and R. G. Voigt, editors, Fifth International Symposium on Domain Decomposition Methods for Partial Differential Equations, pages 19-36, Philadelphia, 1992. SIAM.

[60] H. Wu and Z. Chen. Uniform convergence of multigrid V-cycle on adaptively refined finite element meshes for second order elliptic problems. Science in China: Series A Mathematics, 49(1):1-28, 2006.

[61] J. Xu. Iterative methods by space decomposition and subspace correction. SIAM Rev., 34:581-613, 1992.

[62] J. Xu. The auxiliary space method and optimal multigrid preconditioning techniques for unstructured meshes. Computing, 56:215-235, 1996.

[63] J. Xu. An introduction to multigrid convergence theory. In R. Chan, T. Chan, and G. Golub, editors, Iterative Methods in Scientific Computing. Springer-Verlag, 1997.

[64] J. Xu and L. Zikatanov. The method of alternating projections and the method of subspace corrections in Hilbert space. J. Amer. Math. Soc., 15:573-597, 2002.

[65] H. Yserentant. On the multi-level splitting of finite element spaces. Numer. Math., 49:379-412, 1986.

[66] H. Yserentant. Two preconditioners based on the multi-level splitting of finite element spaces. Numer. Math., 58:163-184, 1990. 